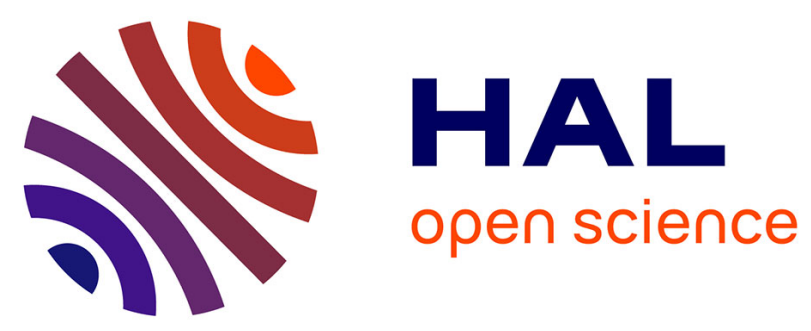

\title{
Numerical FSI Investigation based on LES: Flow past a cylinder with a flexible splitter plate involving large deformations (FSI-PfS-2a)
}

Guillaume de Nayer, Michael Breuer

\section{- To cite this version:}

Guillaume de Nayer, Michael Breuer. Numerical FSI Investigation based on LES: Flow past a cylinder with a flexible splitter plate involving large deformations (FSI-PfS-2a). International Journal of Heat and Fluid Flow, 2014, 50, pp.300-315. 10.1016/j.ijheatfluidflow.2014.08.013 . hal-01086349

\section{HAL Id: hal-01086349 \\ https://hal.science/hal-01086349}

Submitted on 24 Nov 2014

HAL is a multi-disciplinary open access archive for the deposit and dissemination of scientific research documents, whether they are published or not. The documents may come from teaching and research institutions in France or abroad, or from public or private research centers.
L'archive ouverte pluridisciplinaire HAL, est destinée au dépôt et à la diffusion de documents scientifiques de niveau recherche, publiés ou non, émanant des établissements d'enseignement et de recherche français ou étrangers, des laboratoires publics ou privés.

\section{(ㅇ)(1) $\$$}

Distributed under a Creative Commons Attribution - NonCommercial - NoDerivatives $\mid 4.0$ 


\title{
Numerical FSI Investigation based on LES: Flow past a cylinder with a flexible splitter plate involving large deformations (FSI-PfS-2a)
}

\author{
G. De Nayer, M. Breuer* \\ Professur für Strömungsmechanik, Helmut-Schmidt-Universität Hamburg, D-22043 Hamburg, Germany
}

\begin{abstract}
The objective of this paper is to provide a detailed numerical investigation on the fluid-structure interaction (FSI) test case presented in Kalmbach and Breuer (Journal of Fluids and Structures, 42, (2013), pp. 369-387). It relies on detailed experimental investigations on the fluid flow and the structure deformation using modern optical measurement techniques such as particle-image velocimetry and laser triangulation sensors. The present numerical study is based on an efficient partitioned FSI coupling scheme especially developed for turbulent flow simulations around light-weight structures using large-eddy simulation. The current FSI configuration is composed of a fixed cylinder with a flexible thin rubber plate and a rear mass inducing a turbulent flow $(\operatorname{Re}=30,470)$. Mainly based on a movement-induced excitation the flexible structure oscillates in the second swiveling mode involving large deformations. Thus, particular attention has been paid to the computational model and the numerical set-up. Special seven-parameters shell elements are applied to precisely model the flexible structure. Structural tests are carried out to approximate the optimal structural parameters. A fine and smooth fluid mesh has been generated in order to correctly predict the wide range of different flow structures presents near and behind the flexible rubber plate. A phase-averaging is applied to the numerical results obtained, so that they can be compared with the phase-averaged experimental data. Both are found to be in close agreement exhibiting a structure deformation in the second swiveling mode with similar frequencies and amplitudes. Finally, a sensitivity study is carried out to show the influence of different physical parameters (e.g. Young's modulus) and modeling aspects (e.g. subgrid-scale model) on the FSI phenomenon.
\end{abstract}

Keywords: Fluid-structure interaction (FSI); large-eddy simulation (LES); coupled numerical simulation; FSI benchmark; phase-averaging; second swiveling mode

\section{Introduction}

In this paper, a thin flexible structure behind a bluff body in the sub-critical Reynolds number regime is considered. Such a geometrically simple fluid-structure interaction (FSI) problem is useful to validate numerical methods and to investigate how they react on different parameter settings. The long-term objective of the present research project is to simulate practical lightweight structural systems in turbulent flows (textile awnings, outdoor tents, etc.). For this purpose a new numerical FSI simulation methodology using large-eddy simulation (LES) was developed especially for thin flexible structures within turbulent flows [1]. The method was validated at first in laminar flows based on the well-known FSI3 benchmark [2, 3]. The second

${ }^{*}$ Corresponding author

Email address: breuer@hsu-hh.de (M. Breuer) 
step is to test it in turbulent flows requiring a geometrically simple reference test case composed of a thin flexible structure within the turbulent flow regime. A deformable splitter plate clamped behind a bluff body represents on the one hand a geometrically manageable setup. On the other hand complex physical flow features such as separation, transition, and vortex shedding are guaranteed. Hence, it seems to be a good choice. Experimental data are required to evaluate the numerical predictions and to assure their reliability.

A complete review on the topic of thin structures behind bluff bodies was published by Païdoussis [4. Lots of experiments on cantilever plates in axial flow were conducted to investigate the particular instability problem of flutter [5, 6, 7, 8, 9, 10]. Unfortunately, in the experiments presented therein, no flow data are provided. Therefore, these publications cannot be used to completely validate FSI codes. More recently, Gomes and Lienhart [11, 12, 13] have published several FSI test cases including detailed experimental data based on the following geometry: A very thin metal sheet with an additional weight at the end is attached behind a rotating circular cylinder and mounted inside a water channel. The resulting FSI test case was found to be very challenging from the numerical point of view (combination of two-dimensional elements for the thin structure and three-dimensional elements for the rear weight, rotational degree of freedom of the cylinder). Therefore, an additional experimental FSI investigation was carried out based on a slightly different configuration to provide in a first step a less ambitious test case [14]: a fixed cylinder with a thicker rubber tail and without a rear mass is used (test case denoted FSI-PfS-1a). The Reynolds number is set to Re $=30,470$. A complementary numerical investigation of this test case was carried out to show the capabilities of the present FSI code combining LES and FSI [14]. For this first configuration (FSI-PfS-1a) the flexible structure deforms in the first swiveling mode inducing only moderate structural displacements. Good agreement between the experimental and numerical data was achieved. The next step is to take a more challenging test case with large deformations of the plate into account to validate the present numerical methodology. This is the goal of the present study.

For this purpose the geometry used in the previous test case (FSI-PfS-1a) is slightly modified: A $2 \mathrm{~mm}$ thick flexible plate is clamped behind a fixed cylinder. However, this time a rear mass is added at the extremity of the flexible structure, but in contrast to the setup of Gomes and Lienhart [11, 12] the rear mass possesses the same thickness as the rubber plate avoiding a jump in the cross-section. Moreover, the material (para-rubber) is less stiff than in FSI-PfS-1a. Consequently, the flexible structure deforms in the second swiveling mode and the structure deflections are larger than for the first case and completely two-dimensional. The Reynolds number is still $\operatorname{Re}=30,470$. The entire experimental investigations of this test case denoted FSI-PfS-2a are presented in Kalmbach and Breuer [15].

The rear mass and the less stiff material also change completely the governing mechanism responsible for the deformations of the flexible structure. To describe this mechanism and distinguish both test cases, the classification of Naudascher and Rockwell [16] is used. A complex oscillating system involving structures and fluids could exhibit at the same time different types of excitations. In FSI-PfS-1a an instability-induced excitation (IIE) plays the most important role [14]. IIE is provoked by a flow instability which gives rise to flow fluctuations if a specific flow velocity is reached. These fluctuations and the resulting forces become well correlated and their frequency is close to a natural frequency of the flexible structure (lock-in phenomenon). On the contrary, in FSI-PfS-2a a movement-induced excitation (MIE) dominates. MIE is directly linked to body movements and disappears if the body comes to rest. MIE represents a self-excitation: If a body is accelerated in a flow, fluid forces acting on this body are modified by the unsteady flow induced. If a transfer of energy to the moving body appears, a self-excitation is possible, called MIE according to Naudascher and Rockwell [16]. 
This ambitious setup involving large structure deformations and complex flow phenomena is tackled in the present study to further validate the numerical methodology developed and to study the physics of this coupled problem.

The paper is organized as follows: At first, a brief description of the numerical methodology is given in Section 2. Secondly, the test case FSI-PfS-2a is resumed in Section 3 and the full computational setup used is presented. Then, the numerical results are compared with the experimental data in Section 4. Finally, a study on the most important parameters of the test case is carried out in Section 5. All data available for comparison are specified in Section 6 . For the sake of clarity, the investigations on the structural model have been shifted to the Appendix.

\section{Numerical Simulation Methodology}

The numerical method used in the current study relies on an efficient partitioned coupling scheme developed for dynamic fluid-structure interaction problems in turbulent flows. The details of the methodology are published in Breuer et al. 11. Therefore, only a brief summary will be presented here. The FSI code is based on two highly advanced solvers, one for the computational fluid dynamics (CFD) problem and one for the computational structural dynamics (CSD) problem. Between both a coupling program does the required exchange of data.

The fluid solver is an enhanced version of the in-house finite-volume code FASTEST-3D [17, 18]. It is based on a predictor-corrector scheme (projection method) of second-order accuracy in space and time: The momentum equations are advanced in time by an explicit three substeps low-storage Runge-Kutta scheme (predictor step). The predicted velocities are then updated during the correction step, which is based on the solution of a Poisson equation for the pressure correction also updating the pressure. At the end the velocities fulfill the mass conservation equation with a predefined accuracy. The equations are discretized on a curvilinear, blockstructured body-fitted grid with a collocated variable arrangement. The midpoint rule is applied to approximate the surface and volume integrals with second-order accuracy. Furthermore, the flow variables are linearly interpolated to the cell faces leading to a second-order accurate central scheme. The coupling of the pressure and velocity fields on non-staggered grids is assured by the momentum interpolation technique of Rhie and Chow [19]. In the context of FSI the classical Navier-Stokes equations formulated for a fixed domain have to be extended in order to take the deformation of the structure into account. In FASTEST-3D the grid fits to the body. Therefore, the Arbitrary Lagrangian-Eulerian (ALE) formulation is used. The remeshing method is performed algebraically by a combination of linear and transfinite interpolations [20]. Turbulence is taken into account by the LES technique [21]: The large scales in the turbulent flow field are resolved directly, whereas the small scales are modeled either by the Smagorinsky model [22], the dynamic Smagorinsky model according to Germano et al. 23] and Lilly [24] or the WALE model [25].

The structure solver is the finite-element code Carat $++[26$, 27]. This program was developed especially for the prediction of shell or membrane behavior and is based on advanced solution strategies for form finding and non-linear dynamic problems [28, 29, 30]. The momentum equation written in a Lagrangian frame of reference is applied to describe the dynamic equilibrium of the structure. A constitutive relation providing a link between stress and strain is required to close the momentum equation. According to preliminary considerations described in De Nayer et al. [14] a St. Venant-Kirchhoff material law expressed by a second Piola-Kirchhoff stress tensor is assumed. Since in the present case large deformations can occur, the geometry 
is described non-linearly. For the time integration the implicit generalized- $\alpha$ method [31] is used.

To preserve the advantages of the highly adapted CFD and CSD codes and to realize an effective coupling algorithm, a partitioned but nevertheless strong coupling approach is chosen [1]. Since LES typically requires small time steps to resolve the turbulent flow field, the coupling scheme relies on the explicit predictor-corrector scheme forming the kernel of the fluid solver. The code coupling tool used is CoMA [32]. CoMA does the mapping between non-matching grids and the data exchange. For FSI cases the fluid forces and the structural displacements are sent from one solver to the other one. Fluid forces are transferred by a grid-to-grid data interpolation to the CSD code Carat++ using a conservative interpolation scheme [33]. The displacements are bilinearly interpolated [32].

\section{Test case FSI-PfS-2a}

The following section presents at first the FSI test case denoted FSI-PfS-2a. More details on the experiment can be found in [15]. Then, the full computational setup used for all simulations shown in the current paper is presented.

\subsection{Description}

The geometry of the test case is composed of a fixed cylinder, a thin flexible (para-rubber) structure attached to the backside of the cylinder and a rear mass (steel) at its extremity. The steel plate is bonded to the para-rubber material with a waterproofed adhesive for this special material pairing (Loctite 431). The whole structure is installed in a water channel. The blocking ratio is about $9.2 \%$. The geometry of the structure and the test section of the water channel is depicted in Fig. 1 and specified in Table 1. The gravitational acceleration $g$ points in $\mathrm{x}$-direction. Thus in the experimental setup the test section is indeed turned 90 degrees. Several preliminary tests were conducted to find the best working conditions in terms of maximum structure displacements, good reproducibility and measurable structure frequencies within the turbulent flow regime. For this purpose the inflow velocity of the channel $u_{\text {inflow }}$ was varied in a wide range up to about $2.5 \mathrm{~m} / \mathrm{s}$ within the experimental investigation observing the resulting displacements and frequencies of the structure. As shown in Kalmbach and Breuer [15]. For low inflow velocities the flexible structure was found to deform in the first swiveling mode. At an inflow velocity of about $u_{\text {inflow }}=0.9 \mathrm{~m} / \mathrm{s}$ a sudden change in the amplitudes and frequencies of the oscillation was observed leading after a transition process finally to the second swiveling mode. At an inflow velocity $u_{\text {inflow }}$ of $1.385 \mathrm{~m} / \mathrm{s}$ (as for FSI-PfS-1a) nearly symmetrical, large and well reproducible structural displacements were found. This inflow velocity is taken into account for the present test case. Thus, the setup is chosen in such a manner that the inflow velocity guarantees a "stable" oscillation of the flexible structure in the second mode with sufficient distance from the transition regime.

As described in Kalmbach and Breuer [15] the measured inflow turbulence level is low $\left(\mathrm{Tu}_{\text {inflow }}=\right.$ 0.02). The flow parameters are summarized in Table 2. For the structure, the densities were measured and pure structural test cases were performed (see AppendixA) to evaluate the other material parameters (see Table 3). In [15] Young's modulus of the rubber was determined based on a purely structural static test case to $E_{\text {rubber }}=3.15 \mathrm{MPa}$. In the present study a purely structural dynamic test case is carried out and leads to a different value of $E_{\text {rubber }}=4.10 \mathrm{MPa}$. Based on the given inflow velocity and the cylinder diameter the Reynolds number is equal to $\operatorname{Re}=30,470$. The flow around the cylinder is in the so-called sub-critical regime: The boundary layers are still laminar and transition to turbulence occurs in the free shear layers 


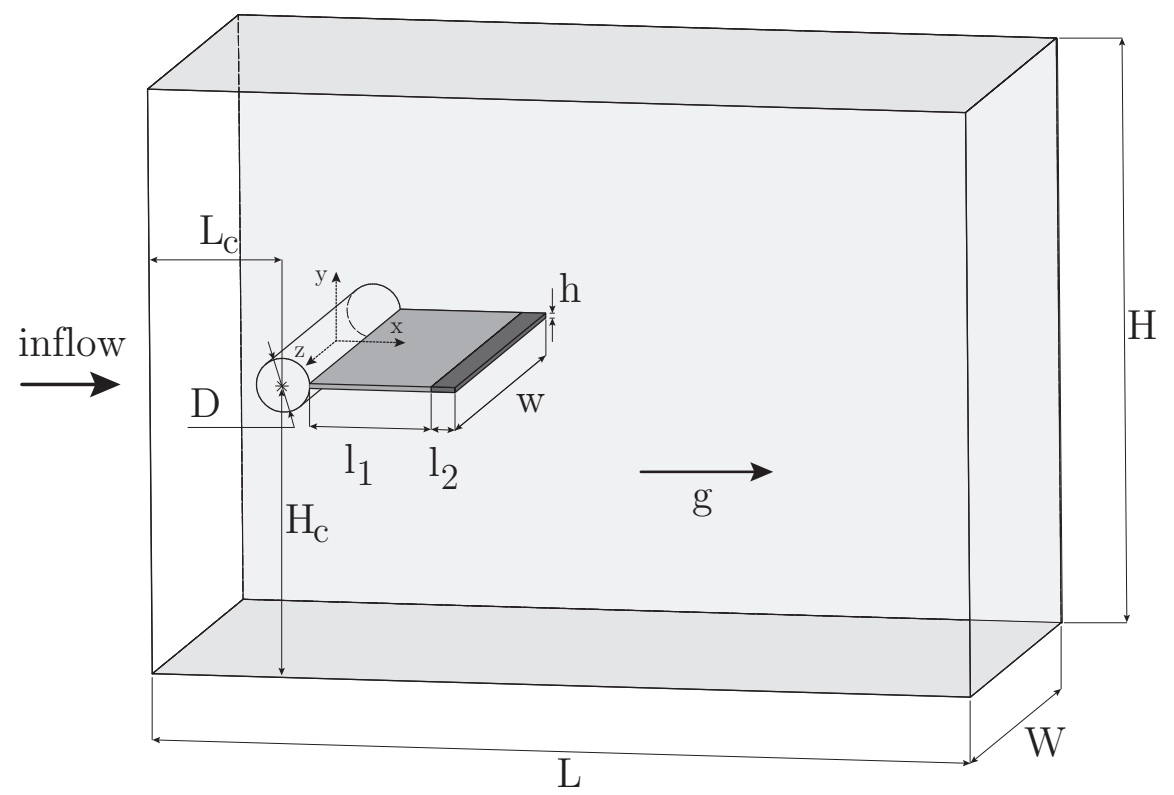

Figure 1: Sketch of the geometrical configuration of the FSI-PfS-2a test case within the test section [15].

Table 1: Geometrical configuration of the FSI-PfS-2a test case.

\begin{tabular}{lll}
\hline Cylinder diameter & $D=0.022 \mathrm{~m}$ & \\
Cylinder center x-position & $L_{c}=0.077 \mathrm{~m}$ & $L_{c} / D=3.5$ \\
Cylinder center y-position & $H_{c}=H / 2=0.120 \mathrm{~m}$ & $H_{c} / D \approx 5.45$ \\
Test section length & $L=0.338 \mathrm{~m}$ & $L / D \approx 15.36$ \\
Test section height & $H=0.240 \mathrm{~m}$ & $H / D \approx 10.91$ \\
Test section width & $W=0.180 \mathrm{~m}$ & $W / D \approx 8.18$ \\
Deformable structure length & $l_{1}=0.050 \mathrm{~m}$ & $l_{1} / D \approx 2.27$ \\
Deformable structure height & $h=0.002 \mathrm{~m}$ & $h / D \approx 0.09$ \\
Deformable structure width & $w=0.177 \mathrm{~m}$ & $w / D \approx 8.05$ \\
rear mass length & $l_{2}=0.010 \mathrm{~m}$ & $l_{2} / D \approx 0.45$ \\
rear mass height & $h=0.002 \mathrm{~m}$ & $h / D \approx 0.09$ \\
rear mass width & $w=0.177 \mathrm{~m}$ & $w / D \approx 8.05$ \\
\hline
\end{tabular}

Table 2: Flow parameters of the FSI-PfS-2a test case.

\begin{tabular}{lll}
\hline Temperature & $\mathrm{T}$ & $=20^{\circ} \mathrm{C}$ \\
Inflow velocity & $u_{\text {inflow }}=1.385 \mathrm{~m} / \mathrm{s}$ \\
Inflow turbulence level & $\mathrm{Tu}_{\text {inflow }}=0.02$ \\
Fluid density & $\rho_{f}=1000 \mathrm{~kg} / \mathrm{m}^{3}$ \\
Dynamic fluid viscosity & $\mu_{f}=1.0 \times 10^{-3} \mathrm{Pas}$ \\
Reynolds number & $\mathrm{Re}=30,470$ \\
\hline
\end{tabular}

evolving from the separated boundary layers behind the apex of the cylinder. Transition to turbulence induces that from that point onwards the flow becomes three-dimensional and chaotic, and consists of a variety of different length and time scales. All these different scales lead to a wide range of frequencies, which are partially visible for example in the fluid forces acting on the structure. The highest frequencies are filtered out by the structure. Only the lowest ones are directly visible in the structural part of the FSI phenomenon: The flexible structure deforms in the second swiveling mode (there are two wave nodes) with a frequency 
Table 3: Structural parameters of the FSI-PfS-2a test case.

\begin{tabular}{ll} 
Flexible structure (para-rubber) & \\
Density & $\rho_{\text {rubber }}=1090 \mathrm{~kg} / \mathrm{m}^{3}$ \\
Young's modulus & $E_{\text {rubber }}=4.1 \mathrm{MPa}$ \\
Poisson's ratio & $\nu_{\text {rubber }}=0.48$ \\
Rear mass (steel) & \\
Density & $\rho_{\text {steel }}=7850 \mathrm{~kg} / \mathrm{m}^{3}$ \\
Young's modulus & $E_{\text {steel }}=210 \times 10^{3} \mathrm{MPa}$ \\
Poisson's ratio & $\nu_{\text {steel }}=0.3$ \\
\hline
\end{tabular}

of $f_{\mathrm{FSI}}=11.25 \mathrm{~Hz}$ (see Fig. 2). The corresponding Strouhal number in the experiment is $\mathrm{St}_{\mathrm{FSI}}=f_{\mathrm{FSI}} D / u_{\text {inflow }}=0.179$.

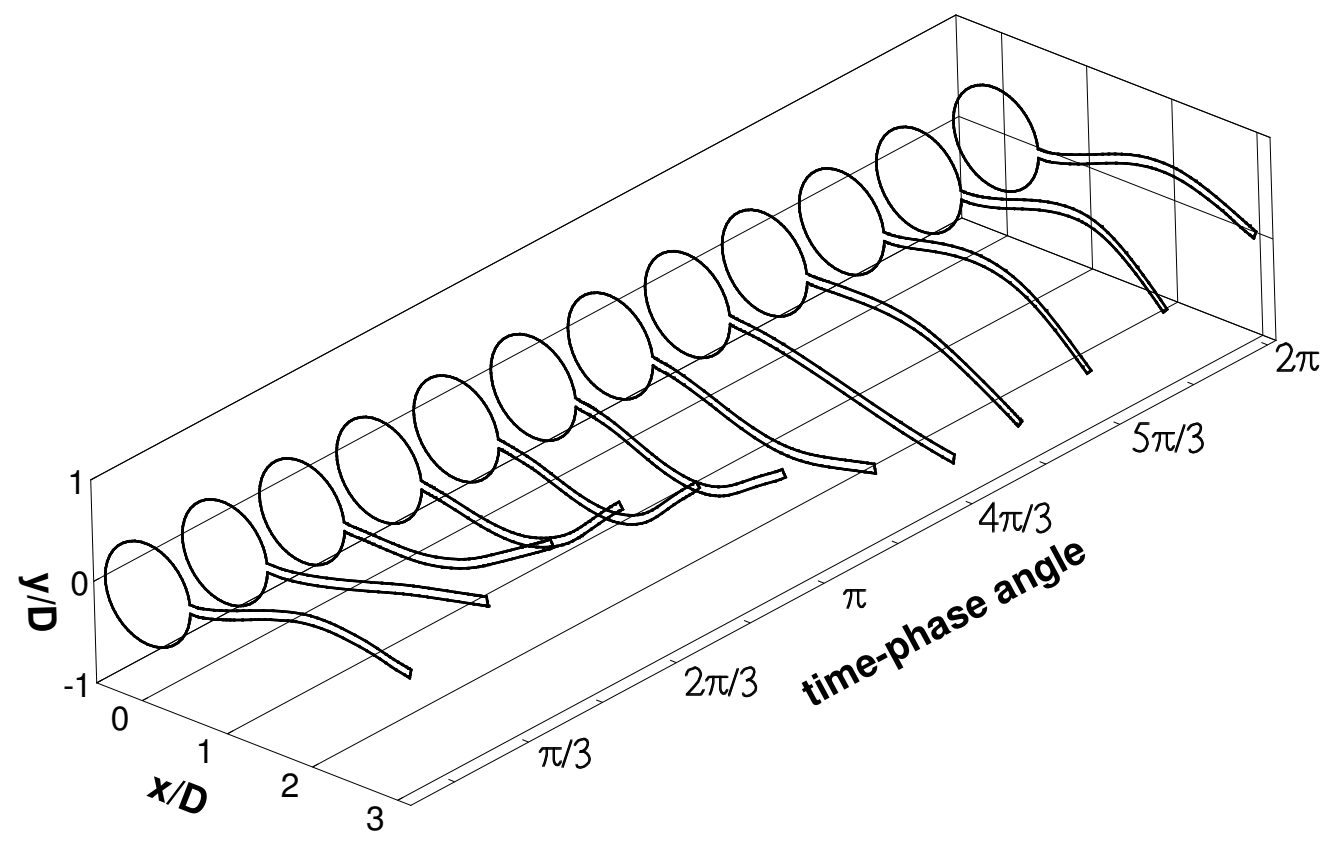

Figure 2: Structure deformation of the reference period in the second swiveling mode, experimental results by Kalmbach and Breuer [15].

\subsection{Computational Setup}

The simulations are based on the numerical methodology summarized in Section 2. In the following the full computational setup for the fluid solver is presented first. Then, the settings for the structure solver are listed. Finally, the options used in the coupling tool will be introduced.

\subsubsection{CFD setup}

Preliminary tests were conducted for the geometrically similar FSI-PfS-1a test case to identify the appropriate CFD domain size [14]. The outcome was that a consideration of a part of the whole test section combined with periodic boundary conditions on the lateral boundaries is sufficient for a mainly two-dimensional structure motion to get reliable flow results. This configuration is denoted subset case. The geometry of the current FSI-PfS-2a test case is similar to FSI-PfS-1a. Owing to the rear mass in the present case avoiding three-dimensional 
deformations of the flexible structure, the restriction to the subset case is even stronger justified than for FSI-PfS-1a. Therefore, the subset case is directly applied here: The depth of the computational domain is not the entire test section width $\mathrm{W} / \mathrm{D} \approx 8.18$, but a subset of the total width equal to the entire length of the splitter plate $\left(l_{1}+l_{2}\right) / \mathrm{D} \approx 2.73$ yielding a quadratic plate.

A block-structured grid is generated consisting of 91 blocks involving about 14 million control volumes $(\mathrm{CVs})$. Note that this resolution is chosen based on the experiences made for the FSIPfS-1a test case [14]. Figure 3 shows the x-y cross-section of the grid. Since only every fourth grid line of the mesh is shown in Fig. 35, the angles between the grid lines and the transitions between the blocks appear to be worse than they are in the full grid. This also holds true for the aspect ratio of the cells in the vicinity of the flexible structure which are in the range between 1 and 8.5. In order to fully resolve the viscous sublayer on the elastic structure, the first cell center is located at a distance of $\Delta y / D \approx 6.8 \times 10^{-4}$ from the flexible structure, which leads to average $y^{+}$values below 0.8 , mostly even below 0.5 near the body. The geometrical stretching factors are kept below 1.1. In spanwise direction the grid consists of 72 equidistant cells. The inflow side is rounded in order to use a C-grid. Consequently, the computational domain in front of the cylinder is slightly larger than in the test section depicted in Fig. 1. At the outlet a convective boundary condition is applied. On the cylinder and on the flexible structure no-slip walls are defined. The top and the bottom of the domain are relatively far away from the flexible structure and are thus taken into account via slip walls. The lateral sides are assumed to be periodic as mentioned above. At the inlet a constant streamwise velocity $u_{\text {inflow }}$ is set as inflow condition without adding any perturbations. The choice of zero turbulence level is based on the consideration that such small perturbations imposed at the inlet will generally not reach the cylinder due to the coarseness of the grid at the outer boundaries. Therefore, all inflow fluctuations will be highly damped. However, since the flow is assumed to be sub-critical and the experimentally determined turbulence level is also small, this disregard is insignificant for the development of the wake and hence the structural motion. This was already proved based on the first test case FSI-PfS-1a [14].

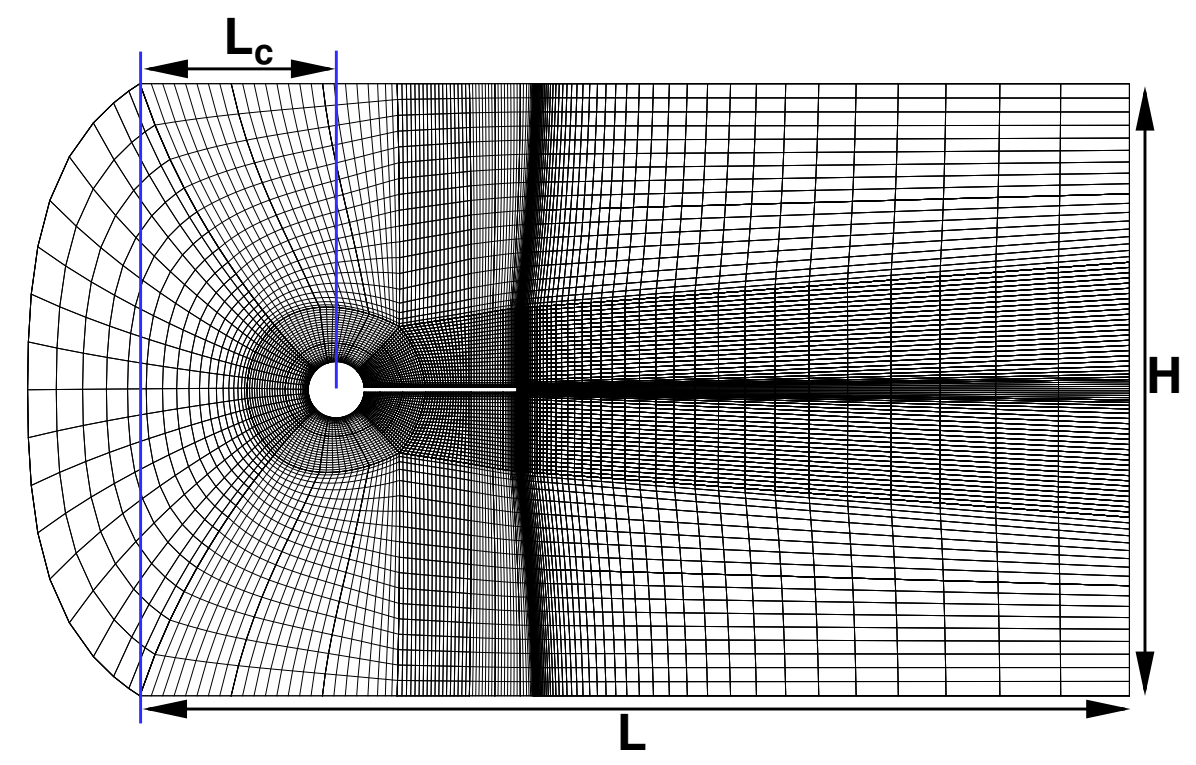

Figure 3: x-y cross-section of the grid used for the simulation with 197,136 CVs (Note that only every fourth grid line in each direction is displayed here).

The numerical method developed is based on an explicit time-marching scheme involving the 
low-storage Runge Kutta algorithm described above. Hence a small time step of $\Delta t=10^{-5} \mathrm{~s}$ is used. This time step size corresponds to a CFL number in the order of unity.

The subgrid-scale (SGS) model chosen for the main simulation is the Smagorinsky model [22]. It is applied with the well established standard constant $C_{s}=0.1$ and a Van Driest damping function near solid walls. Owing to the moderate Reynolds number considered and the fine grid applied, the SGS model is expected to have a limited influence on the results. Nevertheless, in order to investigate and verify this issue, FSI simulations using the dynamic procedure as suggested by Germano et al. [23] and Lilly [24] and the WALE model by Nicoud and Ducros [25] are carried out and analyzed in Section 5 . That includes a study on the model parameters and their influence on the results.

\subsubsection{CSD setup}

The thin flexible structure and the rear mass are modeled by quadrilateral four-nodes shell elements. These elements are special seven-parameters shell elements [34]. Preliminary structural tests show that $30 \times 10$ shell elements for the flexible para-rubber and $5 \times 10$ shell elements for the stainless rear mass are sufficient to deliver accurate results. The well-known Assumed Natural Strain (ANS) [35, 36] is deactivated in the current case and the Enhanced Assumed Strain (EAS) method is set to $(\mathrm{M}, \mathrm{B}, \mathrm{T}, \mathrm{Q}, \mathrm{S})=(4,0,4,4,0)$ avoiding locking phenomena as proposed by Bischoff et al. [37]. A FSI sub-cycling algorithm is not implemented. Therefore, the same time step as for the CFD solver is set.

The flexible structure is clamped to the cylinder: All the nodes at the shell edge in contact with the cylinder have zero degree of freedom (DoF). On the opposite downstream trailing-edge, the rear mass is free to move and all nodes have the full set of six degrees of freedom. The nodes on the lateral sides have to fit to the periodic CFD boundary conditions: The x-displacements, the y-displacements and the rotations have to be the same for a lateral node and its opposite counterpart. Moreover, the periodic boundary conditions imply that the z-displacement of the nodes on the sides are forced to be zero. This special treatment of the lateral nodes is explained in detail and validated in De Nayer et al. [14] for the FSI-PfS-1a test case.

For rubber it is usual to expect a certain level of damping. In the sensitivity study carried out in Section 5 this effect is modeled by a Rayleigh damping (see AppendixA). The damping factor proportional to the stiffness is approximated to a value of $\beta=0.006$, whereas the quantity proportional to the mass $\alpha$ is assumed to be zero. Thus, two additional simulations with Rayleigh damping for two different Young's moduli are carried out within the sensitivity study. Note, however, that based on the outcome of this study, the final numerical reference case used for the comparison with the experimental data is done without Rayleigh damping.

\subsubsection{FSI setup}

In accordance with previous laminar and turbulent test cases in Breuer et al. [1] and De Nayer et al. 114] a linear extrapolation of the structural deformation is used at the beginning of any new time step to accelerate the convergence. A second-order extrapolation is not taken into account, since it was observed that it does not improve the convergence for the current small time step size. Furthermore, a constant underrelaxation factor of $\omega=0.5$ is considered for the displacements during the FSI-subiterations to stabilize the coupling. The forces are exchanged without underrelaxation. The FSI convergence criterion is set to $\varepsilon_{\mathrm{FSI}}=10^{-4}$ for the $\mathrm{L}_{2}$ norm of the displacement differences between two FSI-subiterations normalized by the displacement differences between the current and the old time step. With these settings about 5 FSI-subiterations are required to reach the convergence criterion. Regarding the initial conditions of the coupled simulation, we refer to Section 4 . 
For the sake of clarity, the whole computational setup is summarized in Table 4. The computations are carried out on the national supercomputer SuperMUC: 91 processors are required for the CFD side, one for the CSD program and one for the coupling code. A period of one physical second (i.e., $t^{*}=63$ in dimensionless form) is predicted in about 500 hours wall-clock. The case needs 242 Mbytes per core, which leads to about 22 Gbytes for the entire simulation.

Table 4: Computational setup for FSI-PfS-2a.

\begin{tabular}{|c|c|c|}
\hline \multirow[t]{2}{*}{ CFD setup } & $\begin{array}{l}\text { block-structured mesh } \\
\text { spatial discretization } \\
\text { time discretization } \\
\text { time step } \\
\text { Smagorinsky constant } \\
\text { gravitational acceleration }\end{array}$ & $\begin{array}{l}91 \text { blocks, } 14 \text { million CVs } \\
\text { 2nd order accurate } \\
\text { three substeps low-storage Runge Kutta } \\
\text { (2nd order accurate) } \\
\Delta t=10^{-5} \mathrm{~s} \\
C_{s}=0.1 \\
g_{x}=9.81 \mathrm{~m} / \mathrm{s}^{2}\end{array}$ \\
\hline & $\begin{array}{l}\text { channel inlet } \\
\text { channel outlet } \\
\text { upper channel wall } \\
\text { lower channel wall } \\
\text { lateral sides of the channel }\end{array}$ & $\begin{array}{l}\text { constant streamwise velocity } u_{\infty} \\
\text { convective outflow boundary with } \\
u_{\text {conv }}=u_{\infty} \\
\text { slip wall } \\
\text { slip wall } \\
\text { periodic }\end{array}$ \\
\hline \multirow[t]{2}{*}{ CSD setup } & $\begin{array}{l}\text { flexible structure mesh } \\
\text { rear mass mesh } \\
\text { EAS parameters } \\
\text { ANS method } \\
\text { time discretization } \\
\text { time step } \\
\text { gravitational acceleration }\end{array}$ & $\begin{array}{l}30 \times 10 \text { shell elements } \\
5 \times 10 \text { shell elements } \\
(\mathrm{M}, \mathrm{B}, \mathrm{T}, \mathrm{Q}, \mathrm{S})=(4,0,4,4,0) \\
\text { deactivated } \\
\text { standard Newmark (2nd order accurate) } \\
\Delta t=10^{-5} \mathrm{~s} \\
g_{x}=9.81 \mathrm{~m} / \mathrm{s}^{2}\end{array}$ \\
\hline & $\begin{array}{l}\text { shell edge in contact with the } \\
\text { cylinder } \\
\text { opposite shell edge } \\
\text { lateral sides of the shell }\end{array}$ & $\begin{array}{l}\text { clamped (0 DoF }) \\
\text { totally free ( } 6 \text { DoF }) \\
\text { same x- and y-displacements, } \\
\text { z-displacement imposed to } 0, \\
\text { same rotations }\end{array}$ \\
\hline FSI setup & $\begin{array}{l}\text { predictor (for the structure) } \\
\text { coupling algorithm } \\
\text { convergence limit ( } \mathrm{L}_{2} \text { norm of } \\
\text { the displacement differences) }\end{array}$ & $\begin{array}{l}\text { linear extrapolation } \\
\text { constant underrelaxation on the displace- } \\
\text { ments }(\omega=0.5) \\
\epsilon_{\mathrm{FSI}}=10^{-4}\end{array}$ \\
\hline
\end{tabular}

\section{Comparison between Numerical and Experimental Results}

For the current FSI simulation the flow field is initialized by assuming that the entire structure is non-deformable. For this rigid configuration the turbulent flow is predicted until it reaches a quasi-periodic state. In this case the shell attached to the backside of the cylinder acts like a splitter plate attenuating the generation of a von Kàrmàn vortex street behind the cylinder. Nevertheless, quasi-periodic vortex shedding is still observed with a Strouhal number of $\mathrm{St}_{\text {fixed }} \approx 0.175$. Then, the structure is released and the FSI simulation begins relying on the set-up given in Section 3. A time interval of about 2 seconds physical time is computed. 
The structure requires a few cycles until a statistically steady-state is reached. After that a phase-averaging is carried out.

\subsection{Phase averaging}

The results obtained by the experiment and the simulation cannot be directly compared. Indeed, due to turbulence, the data contain a random chaotic part. This contribution has to be filtered out in order to obtain phase-averaged data which can be compared directly. A special procedure, called phase-averaging method, has been explained in detail in Kalmbach and Breuer [15] to treat both, the experimental and the numerical data sets. For this procedure a representative signal of the FSI problem is needed. In the present case the deformations of the plate are quasi two-dimensional due to the rear mass. Therefore, the y-displacement of the flexible structure at the monitoring point close to the trailing edge depicted in Fig. 4(c) makes sense and is chosen for this purpose. The current FSI phenomenon is quasi-periodic (see Fig. 4(a) and 4(b)]. This quasi-periodicity can also be confirmed by looking at the predicted phase plane depicted in Fig. 4(d) which shows a typical form of a "8" for the second swiveling mode. Furthermore, the history of the y-displacements of the numerical raw results (see Fig. 4(a) and the corresponding experimental data (see Fig. 4(b) are very similar to each other.

Each period found is divided into $n$ equidistant sub-parts (here $n=23$ ). The sub-part $j$ of the period $T_{i}$ corresponds to the sub-part $j$ of the period $T_{i+1}$ and so on. Each data set found in a sub-part $j$ is averaged with the other data sets found in the sub-parts $j$ of all other periods. Finally, data sets of $n=23$ phase-averaged positions for the representative reference period are achieved. For the experiment, about 47 (two-dimensional) particle-image velocimetry (PIV) data sets are averaged for each of the 23 structure positions. For the simulation, about 40 numerical (three-dimensional) data sets are averaged for each of the 23 structure positions. Note that the three-dimensional numerical data sets are also averaged in spanwise direction, in order to get better phase-averaged mean values. Since this is not possible for the twodimensional PIV data sets, it represents the main reason why the phase-averaging procedure for the measurements comprises about 150 periods, whereas for the numerical results only 15 periods are taken into account.

\subsection{Deflection of the structure}

Figure $5(\mathrm{a})$ shows the predicted averaged phase with the standard deviations for each of the 23 structure positions. The maximal standard deviation is about 0.064 and is reached near the phase angle $\phi \approx \pi$. The coefficient of determination $R^{2}$ of the computed mean phase is 0.9925 . The value is very close to unity, which is an indication that the predicted averaged phase is representative for the numerical signal. Figure 5(b) depicts the measured averaged phase with the standard deviations for each of the 23 structure positions. The maximal standard deviation is about 0.072 and is reached just after the phase angle $\phi \approx \pi$. Contrary to the predictions the standard deviation at the maximum is not equal to the one at the minimum.

The averaged phases of the numerical and experimental investigations are compared in Fig. $5(\mathrm{c})$. The maximum of the dimensionless y-displacement $\left.U_{y}^{*}\right|_{\max }=\left.U_{y}\right|_{\max } / D$ is very well predicted with a value of 0.670 . Compared to the measurements $\left(\left.U_{y}^{*}\right|_{\max }=0.667\right)$ a small error of $0.5 \%$ is found. For the minimum of the dimensionless y-displacement $\left.U_{y}^{*}\right|_{\text {min }}=\left.U_{y}\right|_{\text {min }} / D$, the error is larger $(7.2 \%)$, but acceptable. The magnitude of the minimum found in the FSI simulation $\left(\left.U_{y}^{*}\right|_{\min }=-0.674\right)$ is near to the maximum value (0.670). A mean value of -0.002 (see Table 5 ) shows the antisymmetry of the phase with respect to the midpoint $\phi=\pi$. The averaged phase of the measurements is not exactly antisymmetric with respect to $\phi=\pi$ : The minimum is 


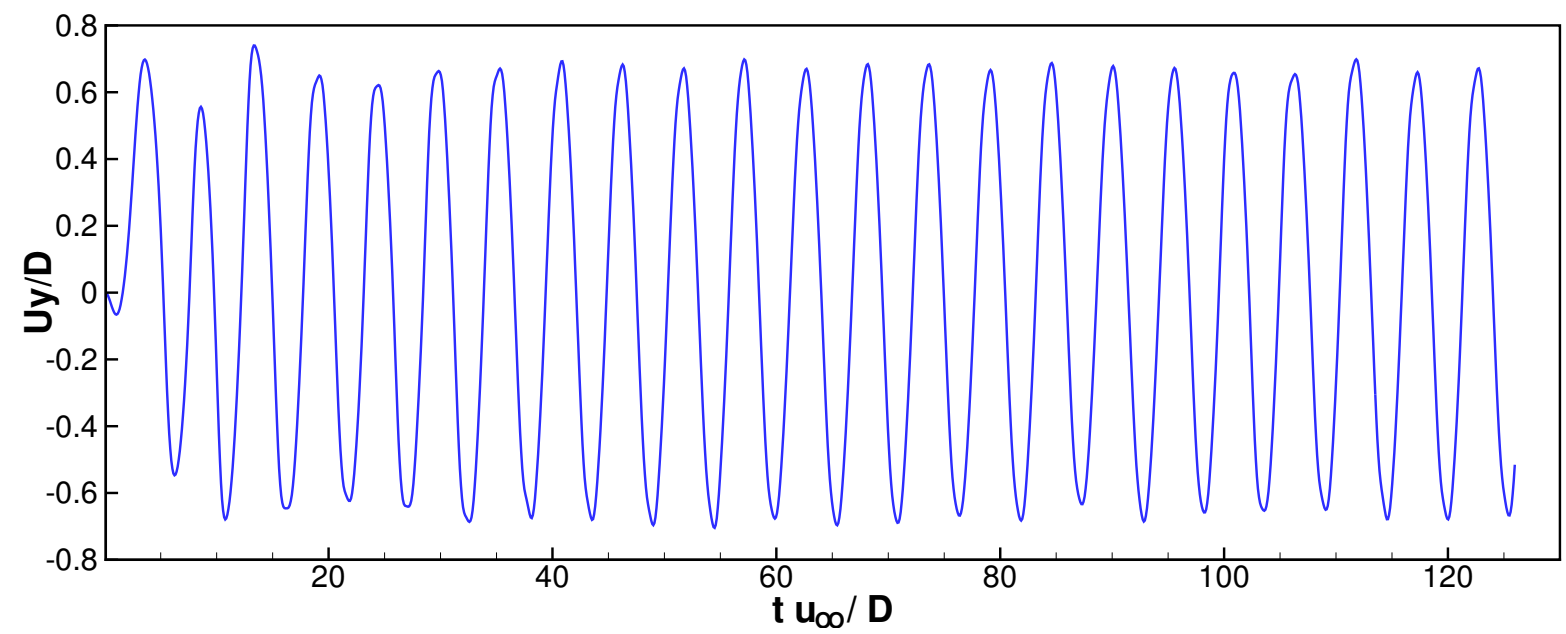

(a) Dimensionless numerical raw y-displacements at the monitoring point sketched below.

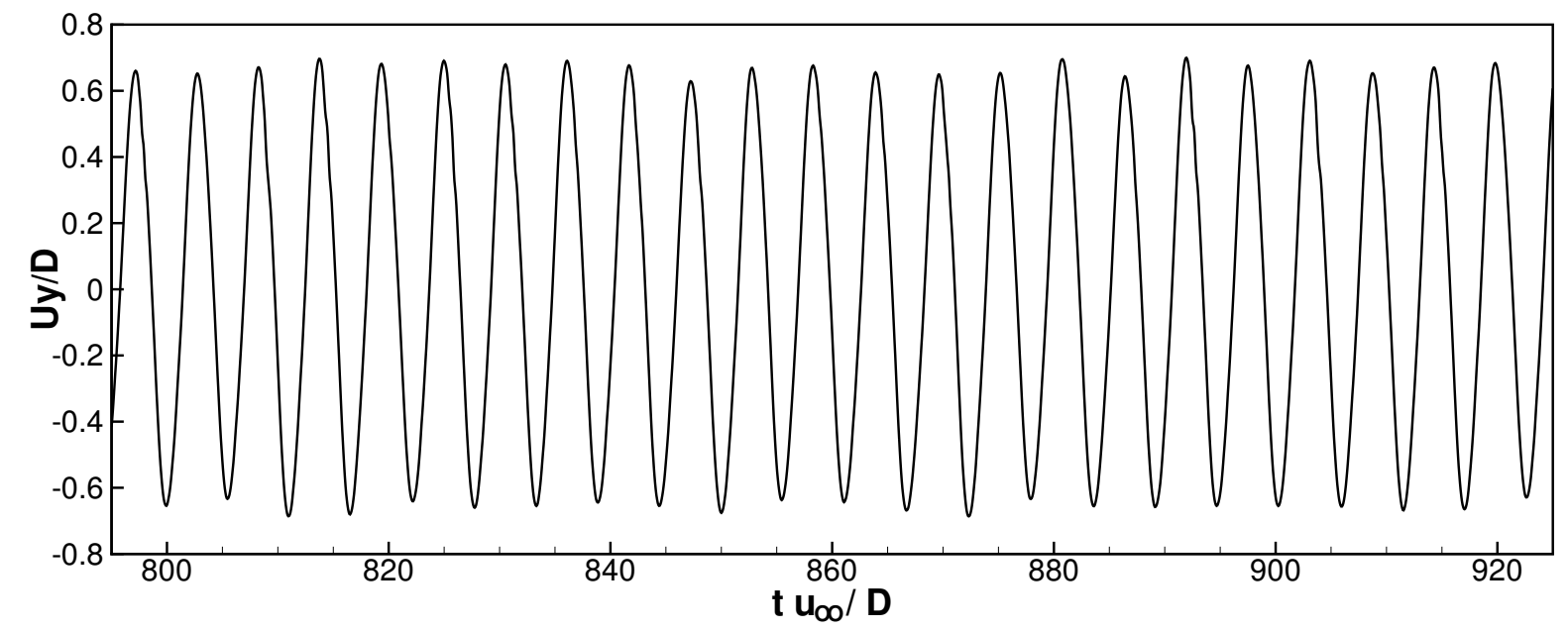

(b) Dimensionless experimental raw y-displacements at the monitoring point sketched below.
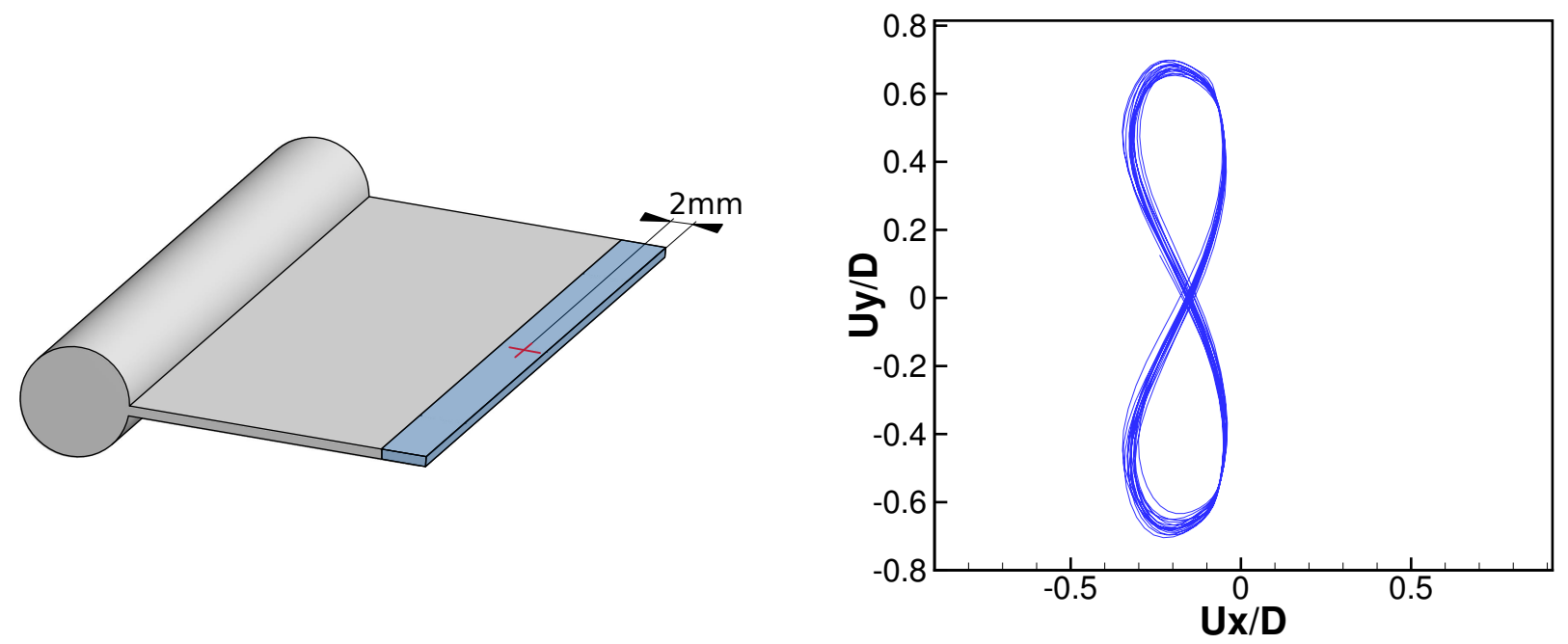

(c) Position of the monitoring point (in red).

(d) Predicted phase plane: $y$-displacement vs. $\mathrm{x}-$ displacement

Figure 4: Numerical and experimental raw signals of dimensionless displacements at a point in the mid-plane of the test section located at a distance of $2 \mathrm{~mm}$ from the shell extremity. 
observed at -0.629 , whereas the maximum is 0.667 yielding an one order of magnitude larger mean value of about 0.019 . The imbalance of the experimental phase can be attributed to minor asymmetries in the experimental setup or in the rubber material and measurement errors due to the laser distance sensor. A careful error analysis led to the conclusion that the major source of errors is related to the assembly of the structure in the test section. That incorporates the bond between the rubber plate and the cylinder and the bond between the cylinder and the test section. Based on uncertainties of only a half degree the observed asymmetry of the measured data can be explained.

Obviously, it is critical to attribute an error of $7.2 \%$ regarding $\left.U_{y}^{*}\right|_{\min }$ (see Table 5 to the predicted data, if the experiment shows a visible asymmetry. Therefore, the amplitude of the oscillations of the structure are also compared in Table 5 and in closer agreement than the extrema showing only a deviation of about 3.7\%. That demonstrate that the error between the predicted and measured data would be much less in case of symmetric experimental data. As written in Section 3.1 the frequency of the FSI phenomenon, i.e., the frequency of the y-displacements, is about $f_{\mathrm{FSI}_{\exp }}=11.25 \mathrm{~Hz}$ in the experimental investigations, which corresponds to a Strouhal number $\mathrm{St}_{\mathrm{FSI}} \underset{\mathrm{exp}}{ } \approx 0.179$. The predicted FSI frequency is $f_{\mathrm{FSI}_{\text {num }}}=11.53 \mathrm{~Hz}$ $\left(\mathrm{St}_{\mathrm{FSI}_{\mathrm{num}}} \approx 0.183\right)$. The error is about $\epsilon_{f}=2.49 \%$. The FSI frequency is also well predicted. A summary of the numerical and experimental values is presented in Table 5 .

Table 5: Comparison between numerical and experimental results.

\begin{tabular}{|l||c|c|c||c|c||c|c||c||c|c|}
\hline \multicolumn{1}{|l||}{ Case } & \multicolumn{10}{|c|}{ Results } \\
\hline & $\begin{array}{c}f_{\mathrm{FSI}} \\
(\mathrm{Hz})\end{array}$ & $\mathrm{St}_{\mathrm{FSI}}$ & $\begin{array}{c}\text { Error } \\
(\%)\end{array}$ & $\left.U_{y}^{*}\right|_{\max }$ & $\begin{array}{c}\text { Error } \\
(\%)\end{array}$ & $\left.U_{y}^{*}\right|_{\min }$ & $\begin{array}{c}\text { Error } \\
(\%)\end{array}$ & Mean value & Amplitude & $\begin{array}{c}\text { Error } \\
(\%)\end{array}$ \\
\hline Simulation & 11.53 & 0.183 & 2.49 & 0.670 & 0.5 & -0.674 & 7.2 & -0.002 & 0.672 & 3.7 \\
\hline Experiment & 11.25 & 0.179 & - & 0.667 & - & -0.629 & - & 0.019 & 0.648 & - \\
\hline
\end{tabular}

\subsection{Flow field}

\subsubsection{Instantaneous flow}

Before comparing the measured and predicted phase-averaged results of the flow, it is interesting to take a look at the raw results. In [15] the authors plot the iso-surfaces of the dimensionless velocity magnitude $|\mathbf{u}| / u_{\text {inflow }}=0.79$ for one given position of the flexible structure. The data were obtained by volumetric three-component velocimetry (V3V) in a volume located behind the flexible structure (see Fig. 6). Figure 7(a) depicts the same iso-surfaces at the same phase angle of the structure $(\phi \approx \pi)$, but based on the numerical data and for the whole computational domain.

In the experiment and the simulation, large quasi-cylindrical structures are visible downstream of the structure. They are consequences of the vortex shedding phenomenon combined with the quasi-periodic motion of the plate. The flow structures observed in the predictions are exhibiting much more small structures than in the experiment. The reason is the resolution of the V3V system $(1940 \times 1940 \mathrm{px})$ which leads to a coarser mesh $(40 \times 40 \times 33$ cells $)$ than in the simulation. This does not allow to resolve the small-scale structures.

Furthermore, the computational domain of the present LES prediction is larger than the measurement volume of the V3V system. Thus, in the simulation the iso-surfaces are also visible near the cylinder and the flexible rubber plate. In this area the structures are very small (see Fig. 7(b)). Due to the motion of the structure the turbulent flow is additionally mixed. In the free shear layers the transition from laminar to turbulent flow occurs earlier than for a rigid 


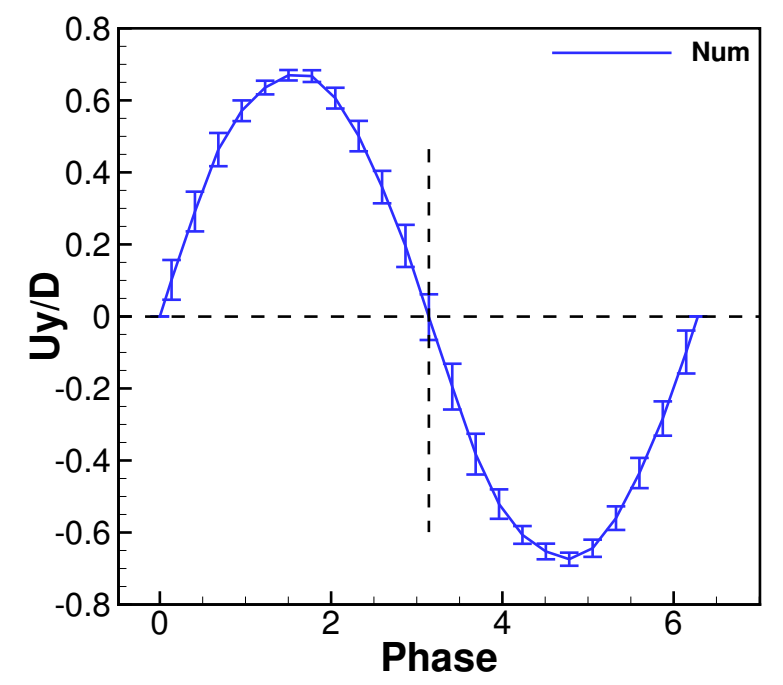

(a) Predicted averaged phase with standard deviations.

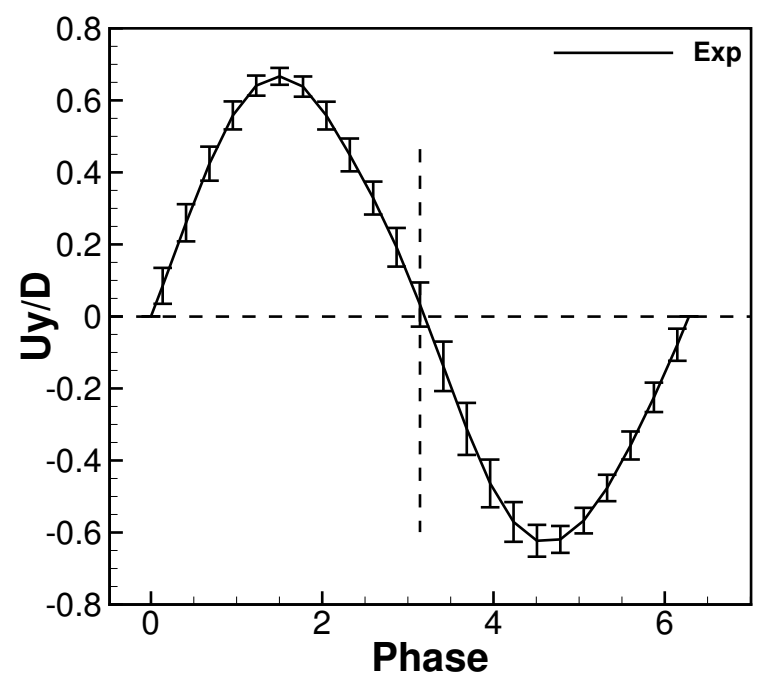

(b) Measured averaged phase with standard deviations.

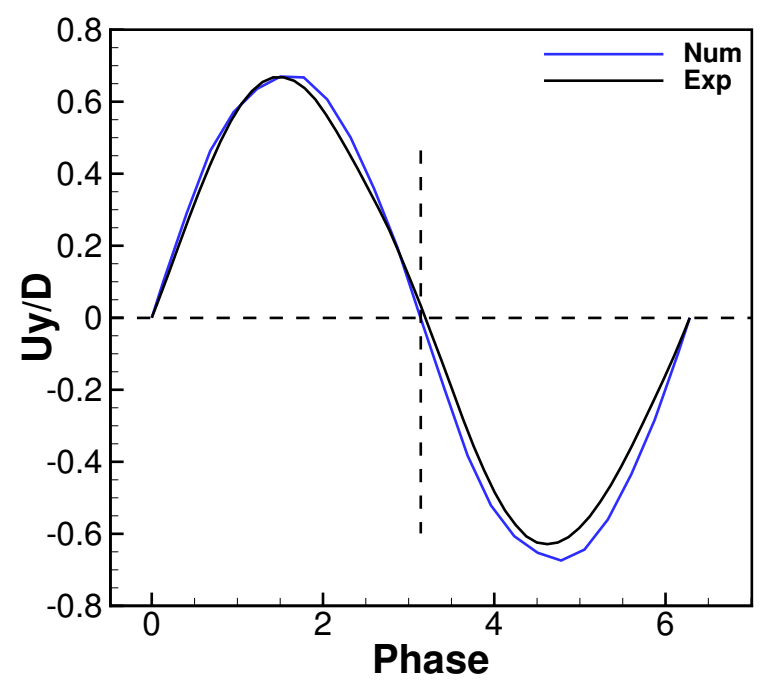

(c) Comparison of the predicted and measured averaged phase.

Figure 5: Numerical and experimental deflections of the structure.

splitter plate fixed behind a cylinder. Indeed, large deformations of the flexible plate induce a stronger backflow, which transports more perturbations into the shear layers and destabilizes them faster (see Fig. 7(c)) .

The iso-surfaces of the velocity magnitude in Fig. 6 and 7(a) are colored by the instantaneous spanwise velocity component. Obviously, spanwise velocities of up to $15 \%$ of the inflow velocity are observed in the wake for both, the experiment and the simulation. Although the structure deformation remains two-dimensional mainly due to the steel rear mass, the flow field is strongly three-dimensional. With the onset of transition in the free shear layers, three-dimensional flow structures start to grow. Owing to the vortex stretching term in the vorticity transport equations the original z-component of the vorticity develops into a full vorticity vector including components in all three directions and thus to a fully three-dimensional flow visible in Fig. 6 and $7(\mathrm{a})$. 


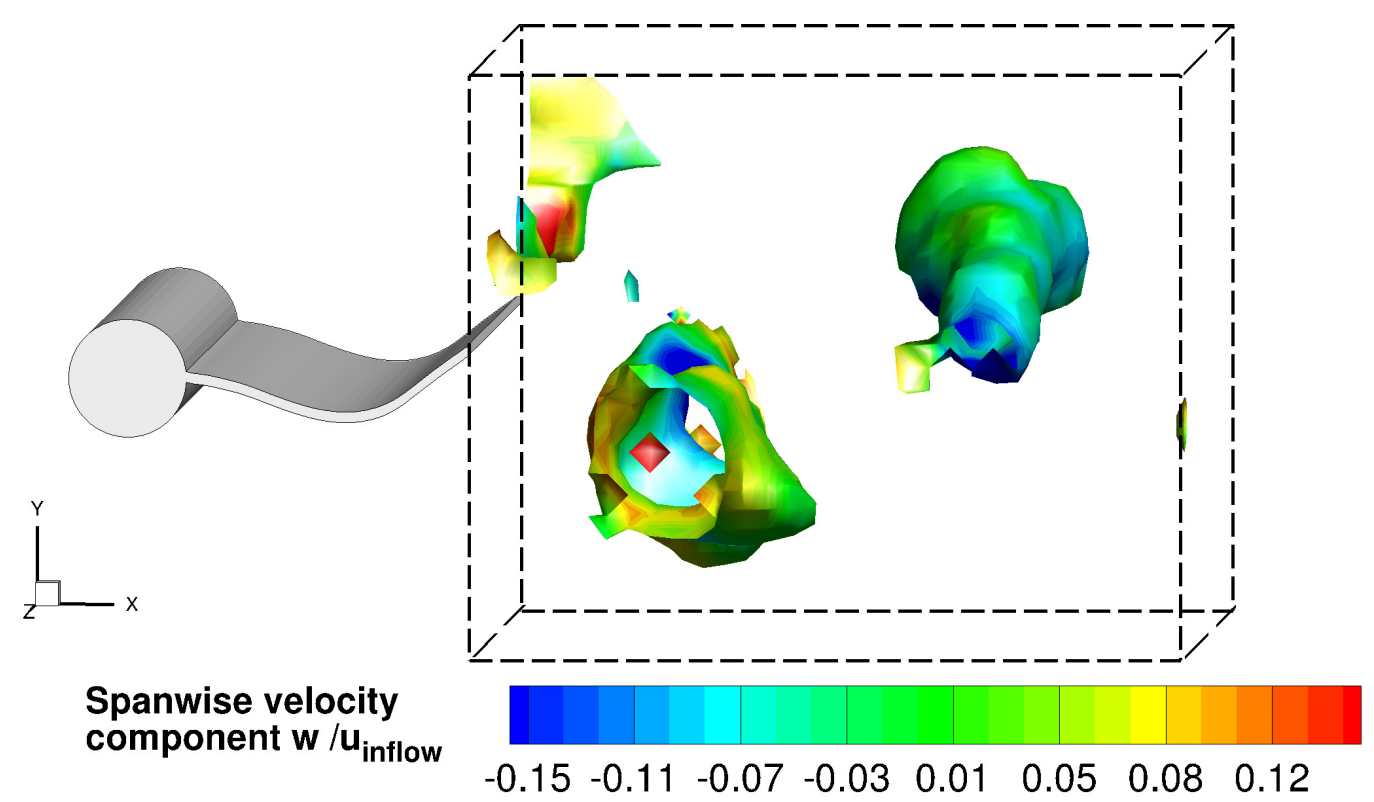

Figure 6: Instantaneous experimental results of the structure and of the flow (V3V) at the phase angle $\phi \approx \pi$ : Iso-surfaces of the instantaneous velocity magnitude $|\mathbf{u}| / u_{\text {inflow }}=0.79$ colored by the instantaneous spanwise velocity component. The box shows the measurement volume.

\subsubsection{Phase-averaged flow}

Applying the phase-averaging procedure on the numerical data, 23 phase-averaged representations of the flexible structure with phase-averaged flow fields are obtained. To describe the whole FSI phenomenon six specific instants were chosen in Kalmbach and Breuer [15]: At $\phi \approx \pi / 12$ the flexible structure moves upwards and its shape is convex. After reaching a maximal upward deflection the structure starts to move in the opposite direction $(\phi \approx 5 \pi / 12)$. The middle of the plate quickly deforms and moves downwards so that the form is now concave $(\phi \approx 9 \pi / 12)$. The middle of the plate continues to move downwards $(\phi \approx 13 \pi / 12)$. The extremity of the flexible structure follows and reaches a maximal downward deformation. Next, the center of the plate displaces upwards again $(\phi \approx 17 \pi / 12)$ and the convex pattern appears again $(\phi \approx 21 \pi / 12)$. The entire cycle is then completed and starts again. Note that the structure possesses two wave nodes and thus exhibits the second swiveling mode as mentioned before.

For the comparison in the present study the streamwise and transverse velocity components are chosen, because they are directly measured in the experiment. Therefore, no indirectly derived quantity such as the vorticity computed based on these velocity components is compared.

Figure 8 compares the experimental and numerical phase-averaged streamwise velocity component for these six phases. For each of the given phase-averaged positions the predicted structure deformations and flow fields are in good agreement with the measurements. The size of the acceleration area and the position of vortices in the wake fit very well between the experiment and the simulation. The shedding phenomenon behind the cylinder is correctly predicted. The computed shear layers are in good agreement with the measured one except in the vicinity of the cylinder. Note that the reasons for the deviations can be explained as will be done below. The recirculation areas found in the present FSI simulation approximately correspond to the experimental observations.

Figure 9 compares the experimental and numerical phase-averaged transverse velocity component only for the first three phases $(\phi \approx\{\pi / 12,5 \pi / 12,9 \pi / 12\})$. Since the FSI phenomenon is antisymmetric (see Fig. 5), the last three phases ( $\phi \approx\{13 \pi / 12,17 \pi / 12,21 \pi / 12\})$ are similar 

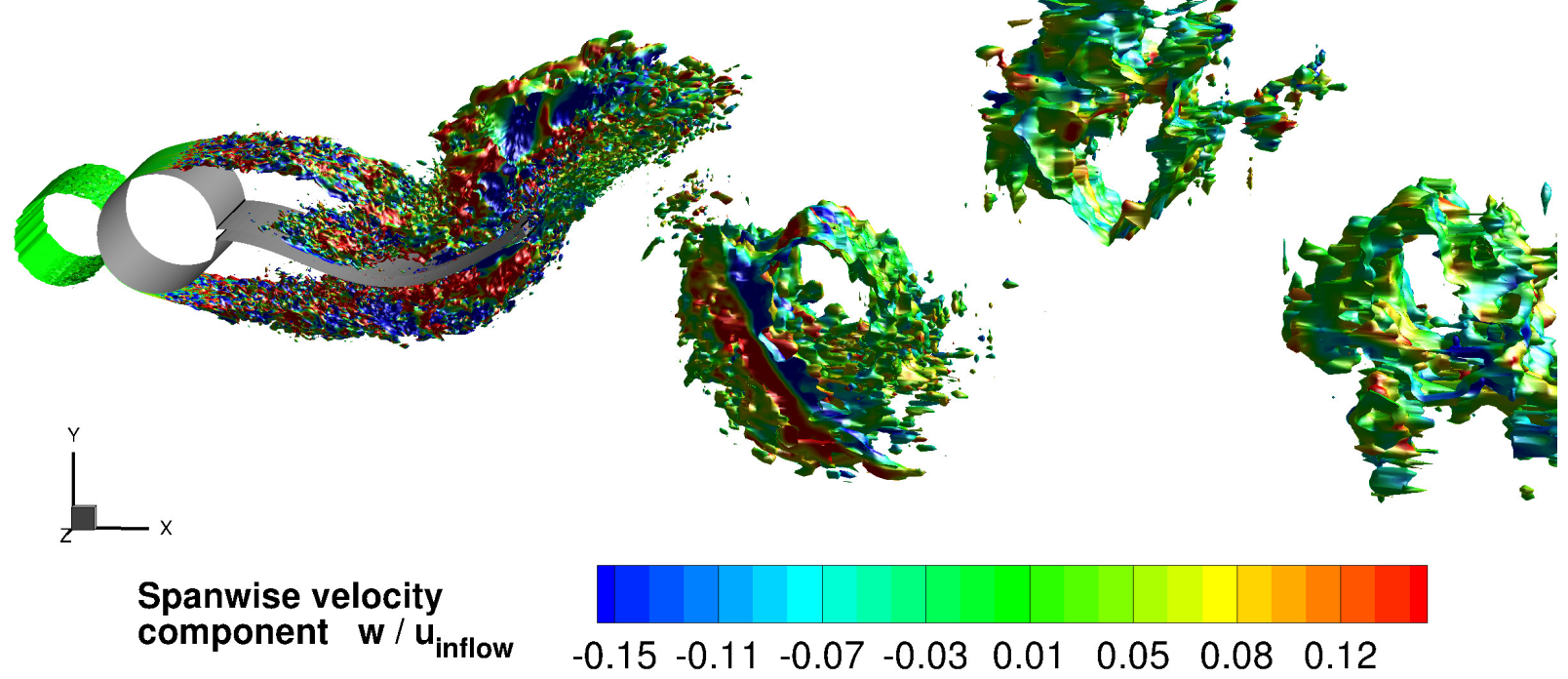

(a) Iso-surfaces of the instantaneous velocity magnitude $|\mathbf{u}| / u_{\text {inflow }}=0.79$; The contours on the iso-surfaces depict the instantaneous spanwise velocity component.

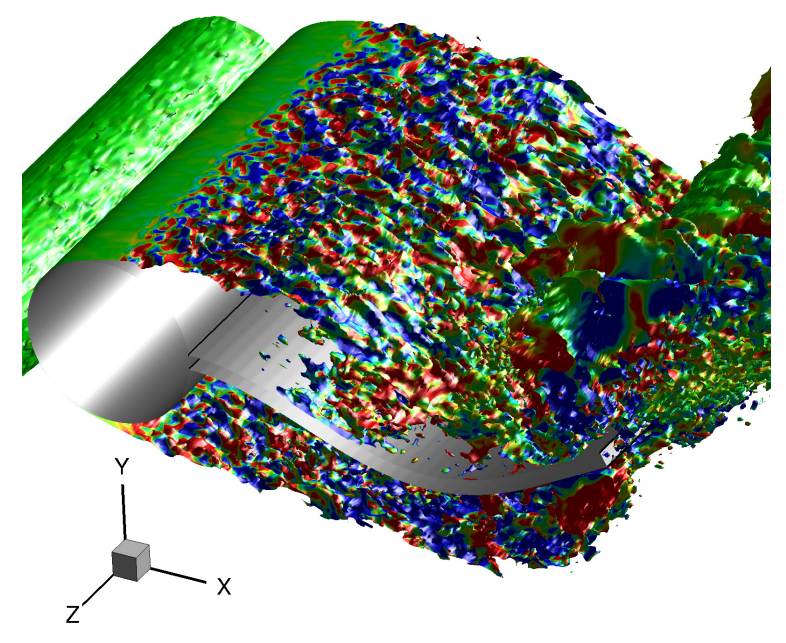

(b) Zoom of the shear layers with the same isosurfaces as in Fig. 7(b),

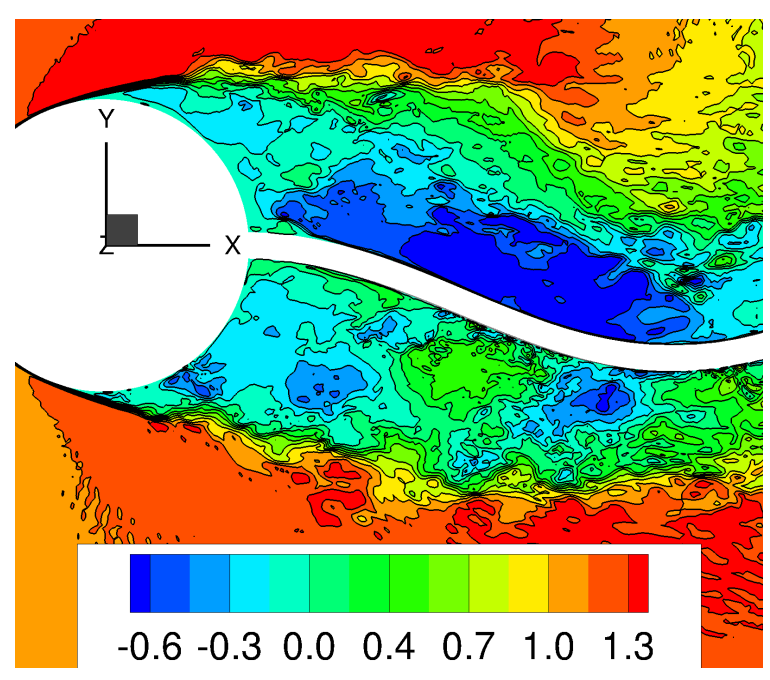

(c) Zoom of the shear layers; The slice represents the dimensionless instantaneous streamwise velocity in the mid-plane.

Figure 7: Instantaneous numerical results of the structure and the flow at the phase angle $\phi \approx \pi$.

to the first three ones and thus can be omitted for the sake of brevity. For all phase-averaged positions the transverse velocity component is in very good agreement with the measured one. Again the predicted sizes and positions of the vortices visible in the contour plots of the transverse velocity component coincide with the measurements.

In order to investigate the predicted results more deeply, the dimensionless absolute error between the simulation and the experiment for two representative positions of the whole FSI phenomenon is visualized in Fig. 10. For both positions the dimensionless absolute local error on the velocity magnitude is mostly below 20\%. The areas with high local errors are located near the structure, in the shear layers and in the zones of maximum velocity of the wake. Three reasons can be found for these differences:

- In the vicinity of the structure, in the shear layers and in the zones of maximum velocity 


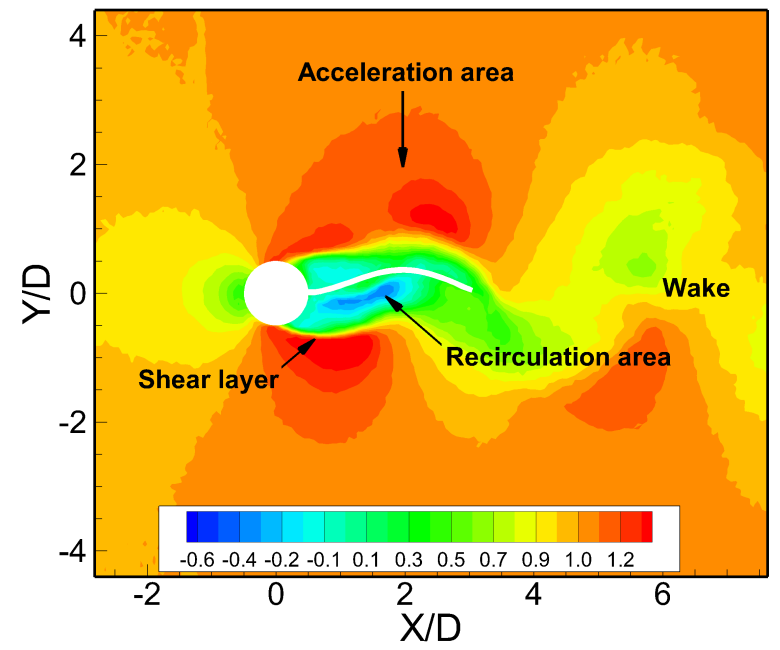

(a) PIV data: $\phi \approx \pi / 12$

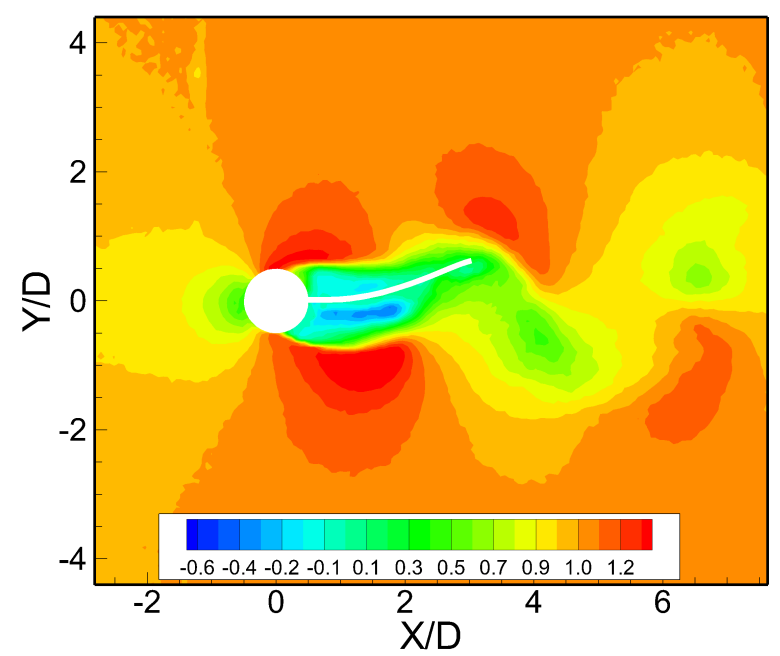

(c) PIV data: $\phi \approx 5 \pi / 12$

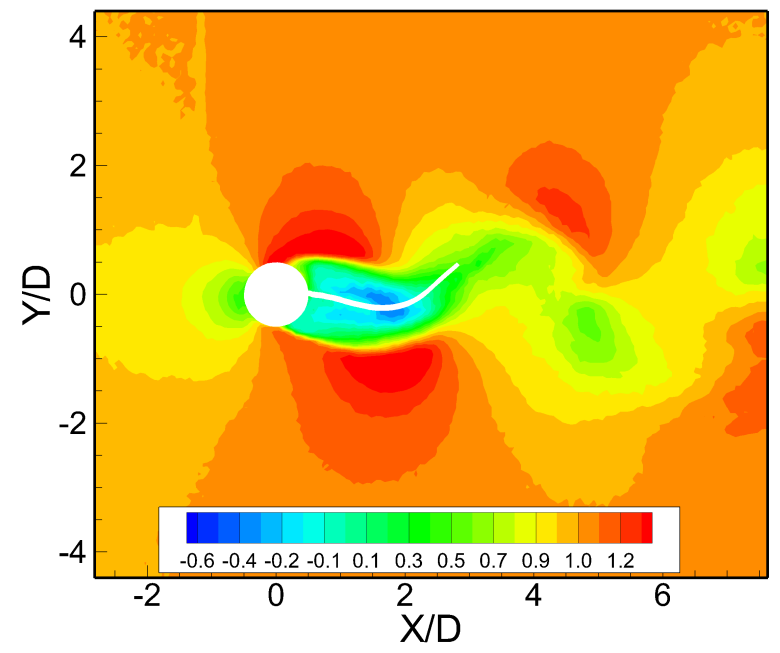

(e) PIV data: $\phi \approx 9 \pi / 12$

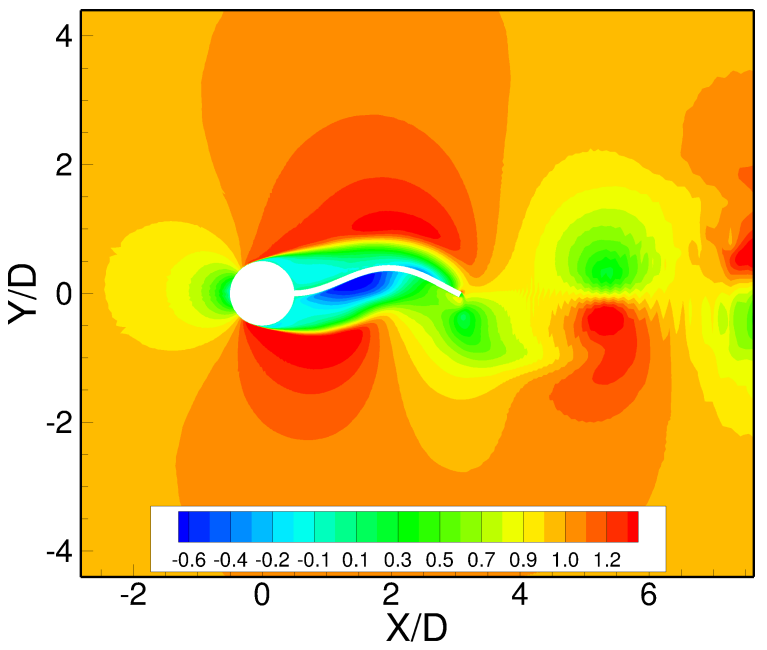

(b) FSI simulation: $\phi \approx \pi / 12$

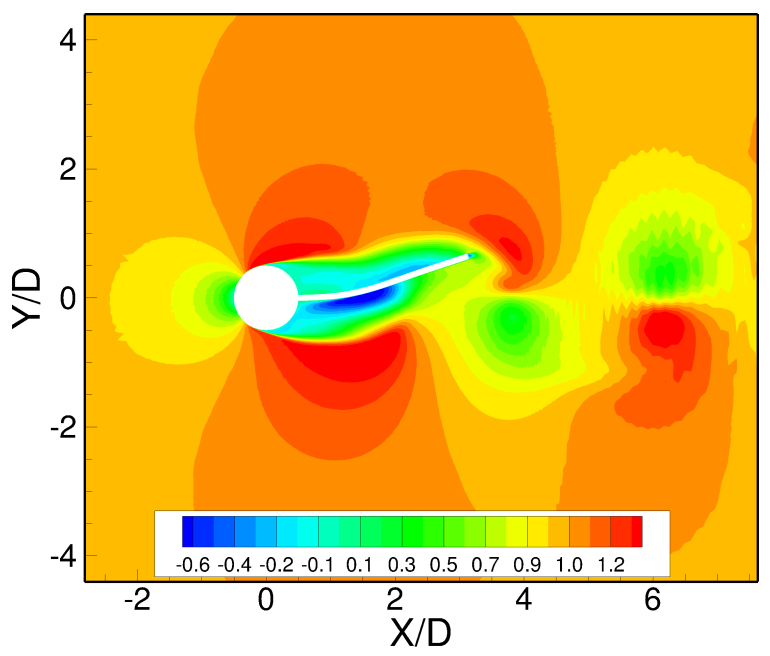

(d) FSI simulation: $\phi \approx 5 \pi / 12$

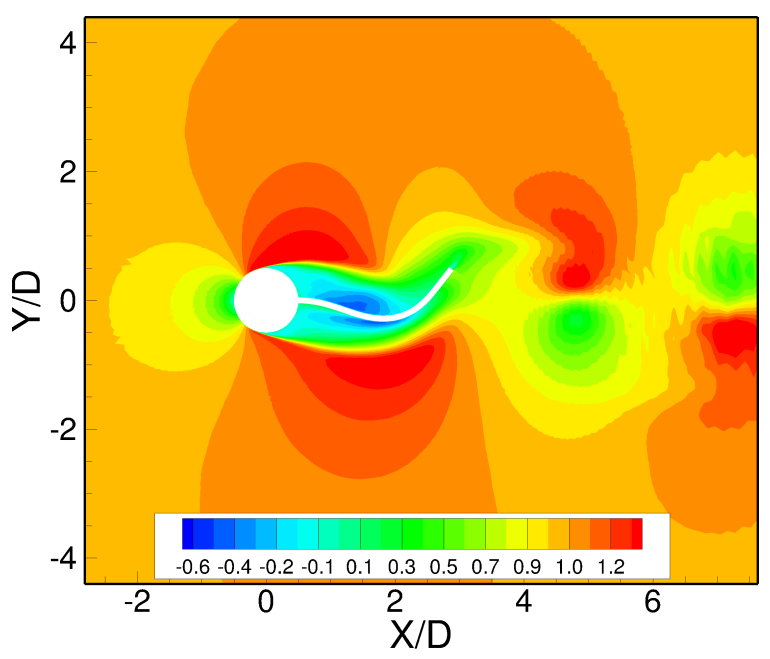

(f) FSI simulation: $\phi \approx 9 \pi / 12$

Figure 8: Comparison of experimental and numerical phase-averaged streamwise velocity component. 


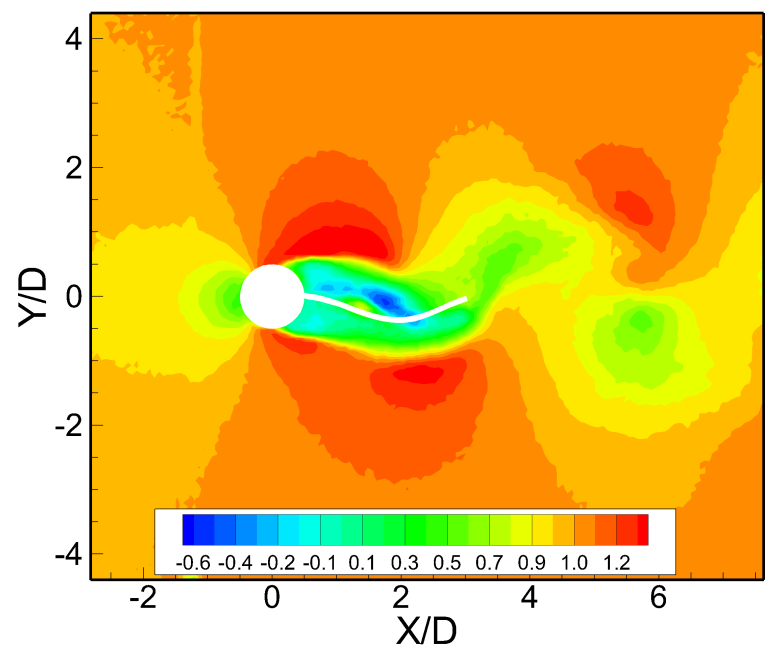

(g) PIV data: $\phi \approx 13 \pi / 12$

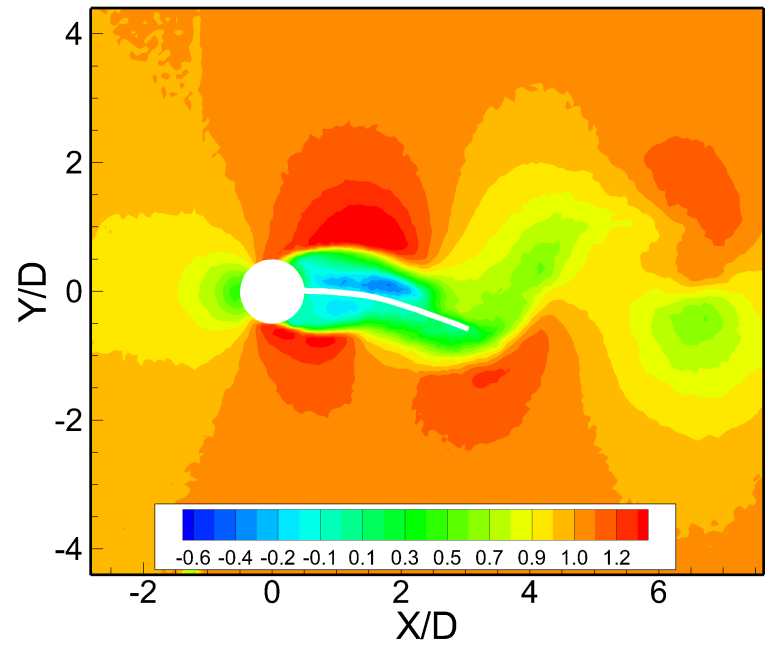

(i) PIV data: $\phi \approx 17 \pi / 12$

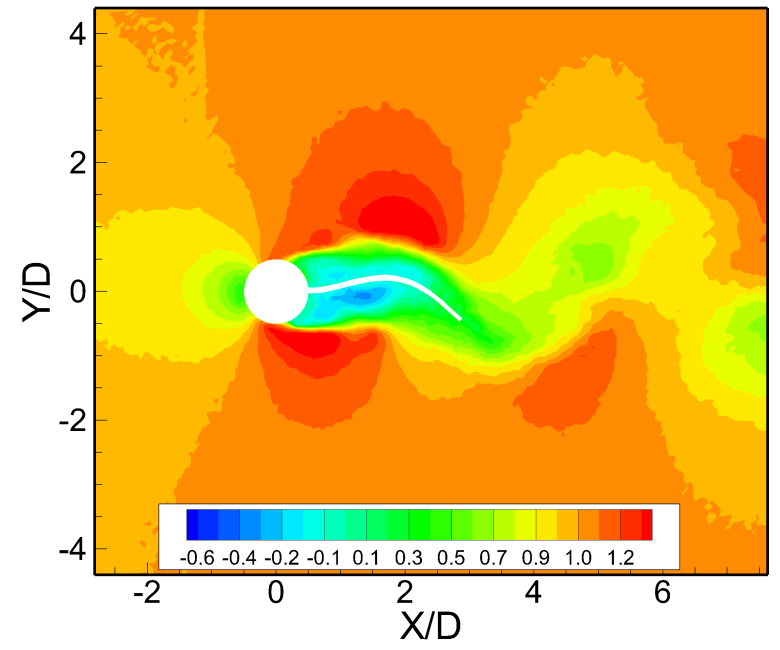

(k) PIV data: $\phi \approx 21 \pi / 12$

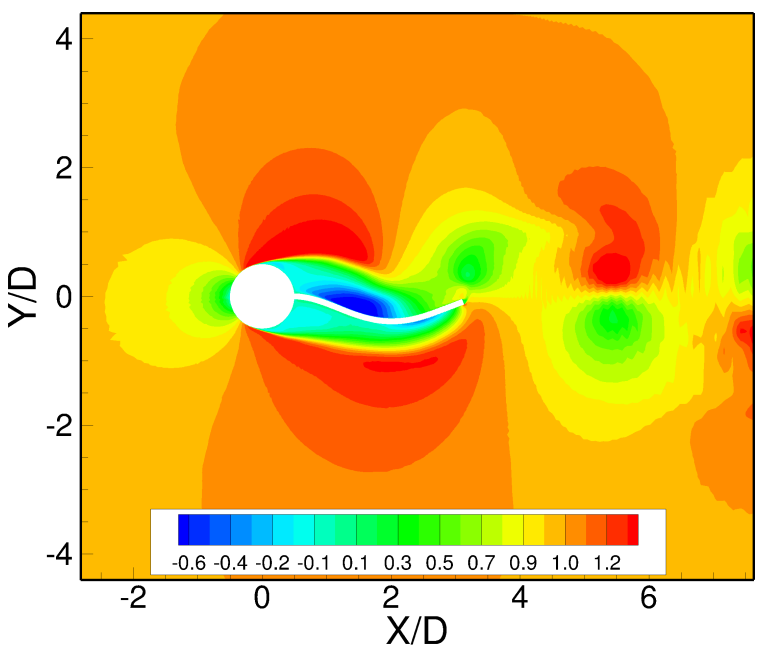

(h) FSI simulation: $\phi \approx 13 \pi / 12$

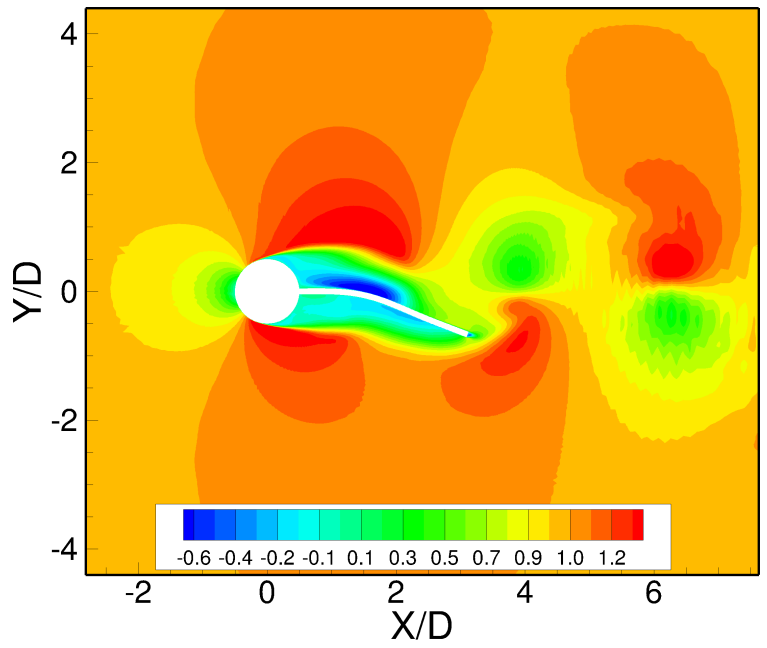

(j) FSI simulation: $\phi \approx 17 \pi / 12$

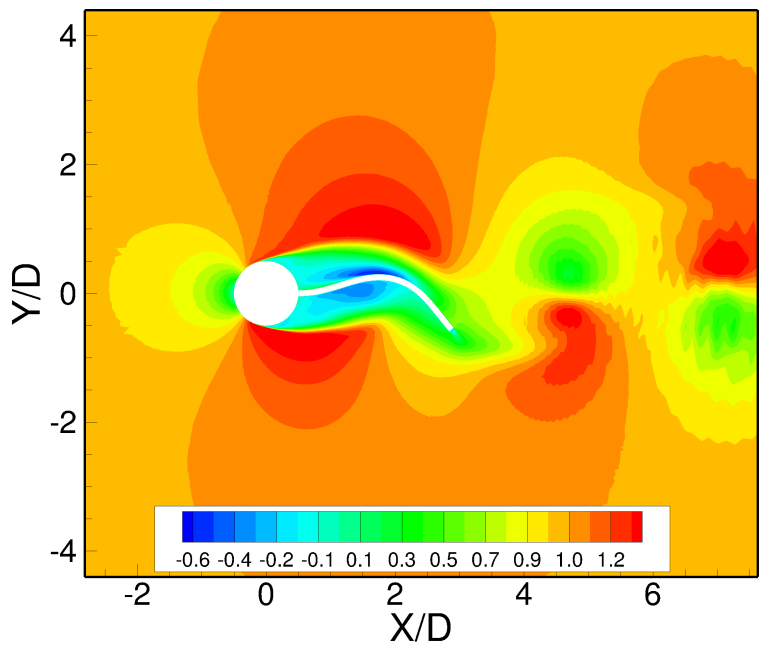

(l) FSI simulation: $\phi \approx 21 \pi / 12$

Figure 8: (continued): Comparison of experimental and numerical phase-averaged streamwise velocity component. 


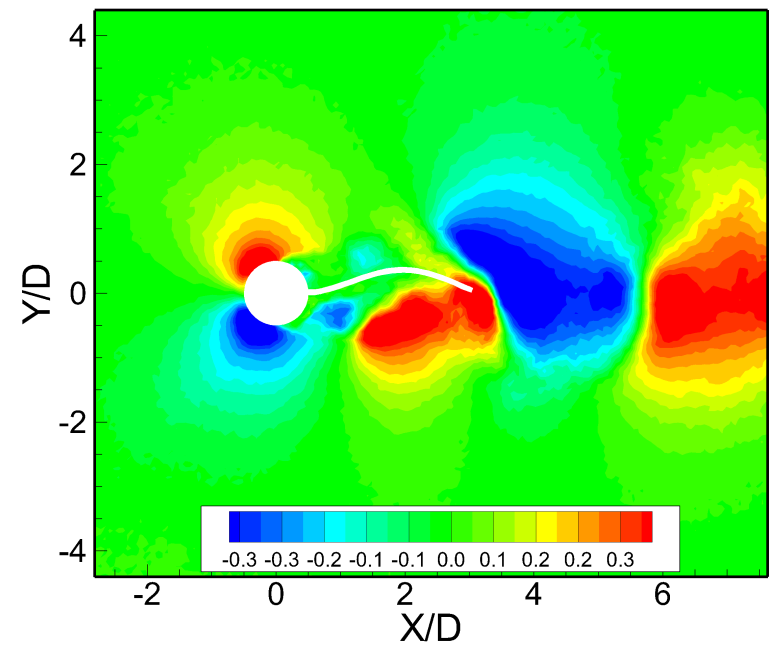

(a) PIV data: $\phi \approx \pi / 12$

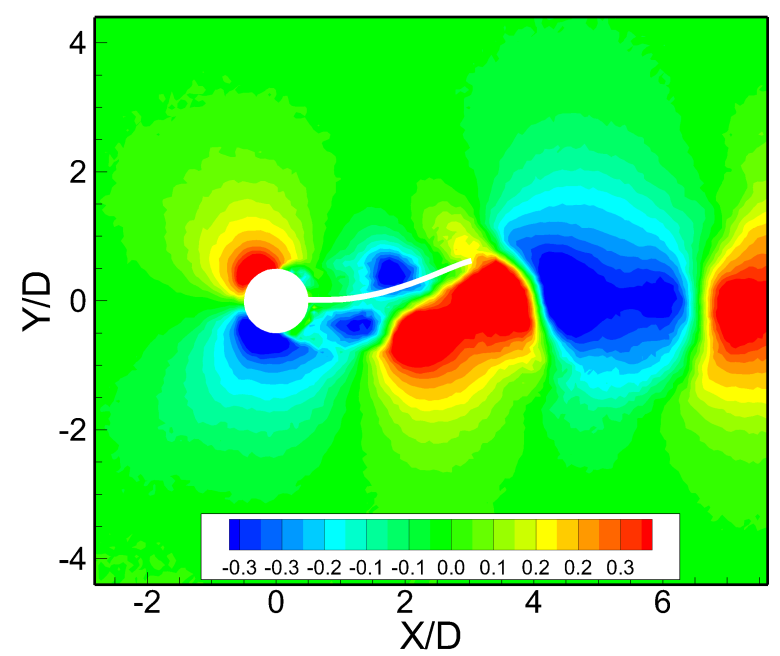

(c) PIV data: $\phi \approx 5 \pi / 12$

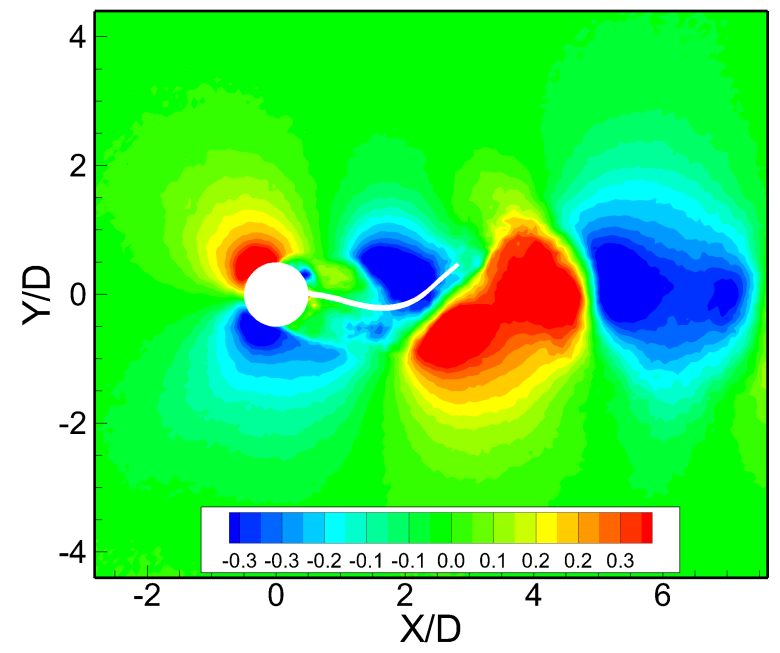

(e) PIV data: $\phi \approx 9 \pi / 12$

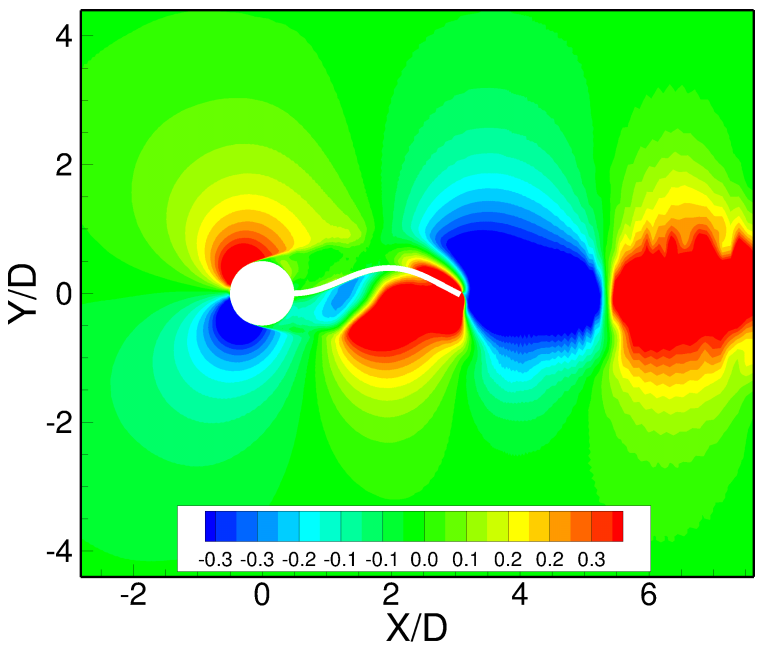

(b) FSI simulation: $\phi \approx \pi / 12$

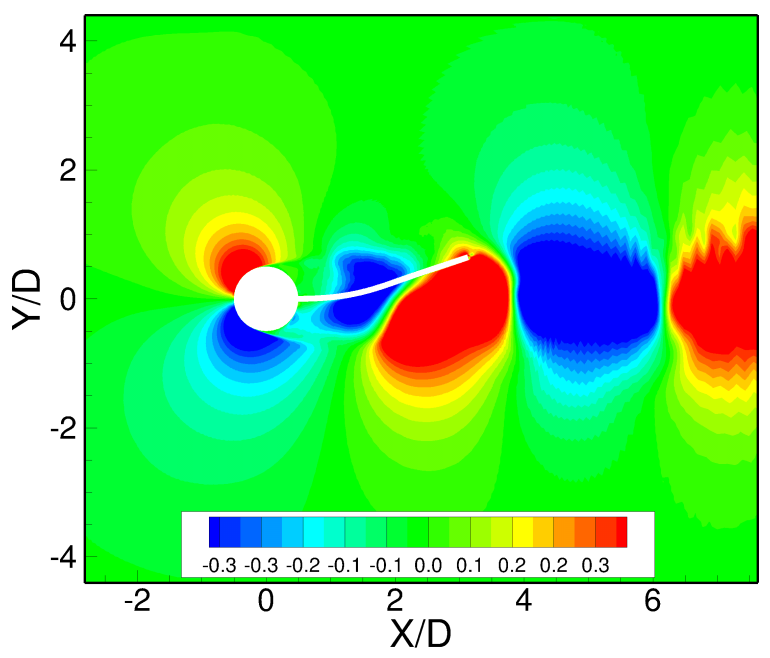

(d) FSI simulation: $\phi \approx 5 \pi / 12$

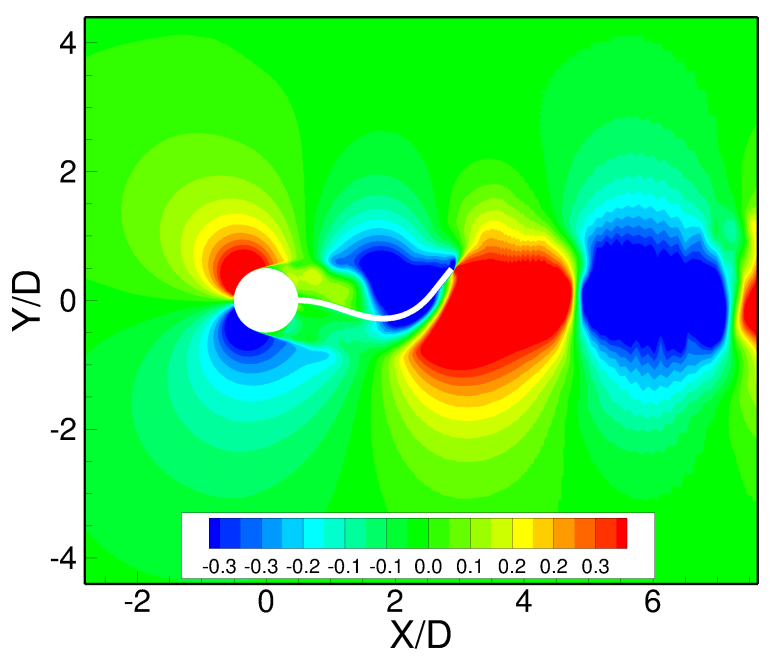

(f) FSI simulation: $\phi \approx 9 \pi / 12$

Figure 9: Comparison of experimental and numerical phase-averaged transverse velocity component. 
the gradients of the flow quantities are large. Since the grid used for the simulation is much finer than the PIV measurement mesh, the accuracy of the numerical solution is much higher than the precision of the PIV measurements in these regions.

- Reflections of the laser light at the surface of the cylinder lead to inaccuracies of the PIV measurements in the proximity of the structure.

- The measurement error is more important for low flow velocities since the uncertainties for the velocity expected by the PIV method is calculated to about $0.085 \mathrm{~m} / \mathrm{s}$ [38]. The areas exhibiting higher errors are surrounding the cylinder and the rubber plate, where the flow velocity is small.

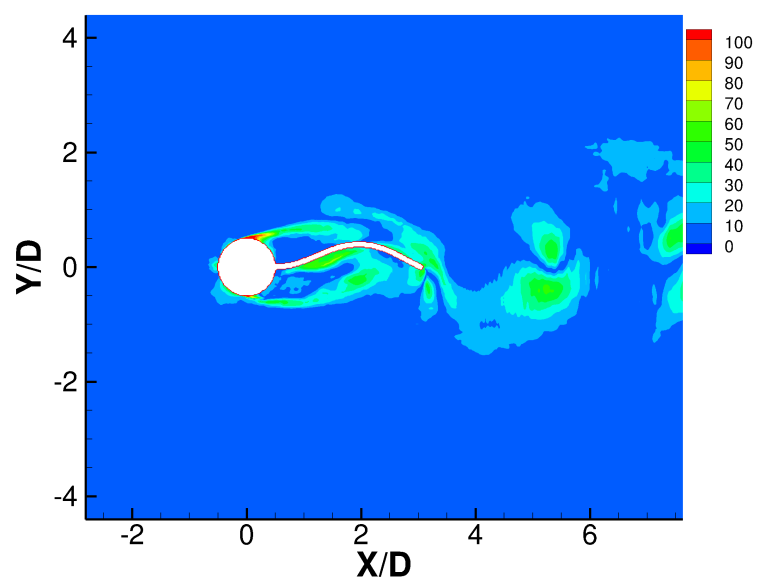

(a) $\phi \approx \pi / 12$.

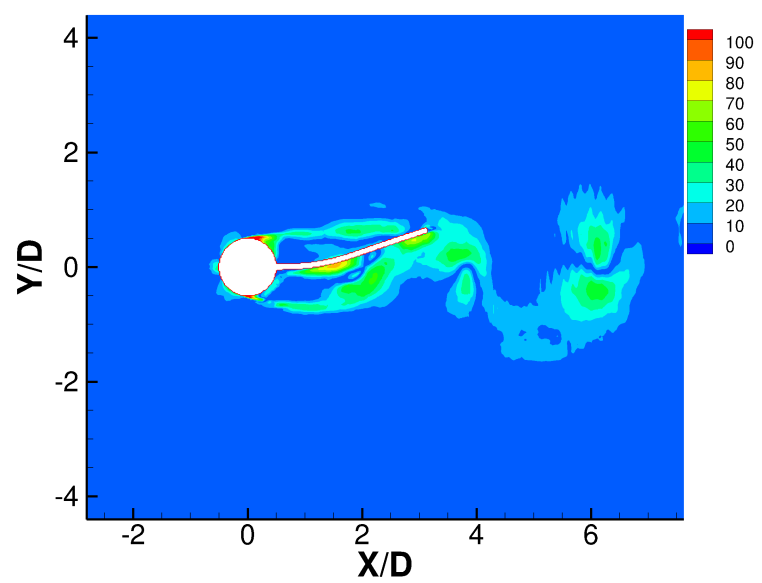

(b) $\phi \approx 5 \pi / 12$.

Figure 10: Dimensionless absolute local error (velocity magnitude) in \%.

In summary, larger local errors are only appearing at locations, where the measurement technique applied has certain deficits. Despite these local differences the computed flow is in very good agreement with the measured one for each of the six positions shown and hence for the entire period.

\section{Sensitivity Study}

In order to better understand the current test case a dimensional analysis was carried out. The physical quantities of the present FSI problem are:

- For the fluid: The dynamic viscosity $\mu_{f}$, the fluid density $\rho_{f}$, the inlet velocity $u_{\text {inflow}}$;

- For the geometry: The cylinder diameter $D$, the dimensions of the rubber plate and of the rear mass $l_{1}, l_{2}, w$ and $h$;

- For the flexible structure: The Young's modulus $E_{\text {rubber }}$, the Poisson's ratio $\nu_{\text {rubber }}$ and the density $\rho_{\text {rubber. }}$.

- For the description of the FSI phenomenon: The FSI frequency $f_{\mathrm{FSI}}$ and the displacement extrema $\left.U_{y}\right|_{\max }$ and $\left.U_{y}\right|_{\min }$. 
The physical quantities of the rigid structures are not taken into account in this dimensional analysis.

These 14 physical quantities lead to 11 dimensionless parameters:

- The Reynolds number $\operatorname{Re}=\rho_{f} u_{\text {inflow }} D / \mu_{f}$ for the fluid;

- The length ratios $w / D, h / D, l_{1} / D$ and $l_{2} / D$ for the geometry;

- $\nu_{\text {rubber }}$ for the material of the rubber plate;

- The density ratio $\rho_{f} / \rho_{\text {rubber }}$, the Cauchy number $\mathrm{Cy}_{\text {rubber }}=\rho_{f} u_{\text {inflow }}^{2} / E_{\text {rubber }}$ [39], the extrema of the dimensionless y-displacements $\left.U_{y}^{*}\right|_{\max }=\left.U_{y}\right|_{\max } / D$ and $\left.U_{y}^{*}\right|_{\min }=\left.U_{y}\right|_{\min } / D$ and the Strouhal number $\mathrm{St}_{\mathrm{FSI}}=f_{\mathrm{FSI}} D / u_{\text {inflow }}$ for the FSI coupling.

In the present experimental investigation the operating conditions $\left(\mu_{f}, \rho_{f}\right.$ and $\left.u_{\text {inflow }}\right)$ for the fluid are well-known and fixed. The dimensions of the cylinder, of the rubber plate and of the rear mass are well defined, too. Therefore, the Reynolds number Re and all geometrical ratios $w / D, h / D, l_{1} / D$ and $l_{2} / D$ are fixed in the sensitivity study. Because the dimensions of the structures are well defined, the density $\rho_{\text {rubber }}$ is precisely measured. The density ratio $\rho_{f} / \rho_{\text {rubber }}$ is also fixed. The Poisson's ratio of the para-rubber is $\nu_{\text {rubber }}=0.5$. However, for numerical reasons it has to be set to a slightly different value to avoid dividing by zero (here $\left.\nu_{\text {rubber }}=0.48\right)$. Consequently, seven of the eleven dimensionless parameters are already fixed. On the contrary, Young's modulus of the rubber plate $E_{\text {rubber }}$ is determined by purely structural tests (see AppendixA). It has an important influence on the modeling of the material [14]. Therefore, several values for $E_{\text {rubber }}$ and consequently of $\mathrm{Cy}_{\text {rubber }}$ are tested to evaluate this influence. The value $E_{\text {rubber }}=3.15 \mathrm{MPa}$ is mostly used, because it is the Young's modulus given in [15]. $E_{\text {rubber }}=4.10 \mathrm{MPa}$ is the approximated value found by the dynamic structural test (see AppendixA). The other ones are arbitrarily selected close to the values mentioned above.

In summary, the dimensional analysis presented here is restricted to the four following dimensionless numbers:

- the Cauchy number $\mathrm{Cy}_{\text {rubber }}$,

- the dimensionless extrema $\left.U_{y}^{*}\right|_{\max }$ and $\left.U_{y}^{*}\right|_{\min }$ of the y-displacement and

- the Strouhal number $\mathrm{St}_{\mathrm{FSI}}$.

However, since it is a simulation, modeling aspects are of course significant and have to be taken into account in the sensitivity study.

The present test case is within the turbulent regime. Hence, turbulence plays an important role. The modeled part of the LES method is based on the subgrid-scale (SGS) model. The parameter of the chosen model has to be set in a range of values: For example for the Smagorinsky model reasonable parameters in the range $C_{s} \in[0.065,0.2]$ are found in the literature. Consequently, simulations with different SGS models and different parameter values are carried out. Three simulations using the Smagorinsky model are presented: the Smagorinsky constant is adjusted to test the whole range $\left(C_{s}=0.065,0.1\right.$ and 0.18$)$. Furthermore, a simulation with the dynamic procedure of Germano et al. [23] still relying on the Smagorinsky model is carried out. Finally, the WALE model is tested with a $C_{W}=0.33$, corresponding to a Smagorinsky constant of $C_{s}=0.1$. 
As already explained, the modeling of the material has a strong influence on the coupled simulation. As explained in Section 3.2.2 for rubber it is usual to expect a certain level of damping which is modeled by a Rayleigh damping (see AppendixA) with $\beta=0.006$ and $\alpha=0$. Thus, two simulations with Rayleigh damping for two Young's moduli are also carried out and complete the sensitivity study.

All tests are summarized in Table 6. The experimental results are also added as the reference. Each simulation was done for a time interval of $2 \mathrm{~s}$ physical time and comprises about 15 swiveling periods without the initial starting phase. Relative errors between the numerical and experimental values are given.

The following results and trends can be seen concerning the influence of the Rayleigh damping:

- For both Young's moduli, $E_{\text {rubber }}=3.15 \mathrm{MPa}$ and $4.10 \mathrm{MPa}$, the frequencies $f_{\mathrm{FSI}}$ and consequently the Strouhal numbers $\mathrm{St}_{\mathrm{FSI}}$ are slightly lower for the simulations with structural damping than for the simulations without.

- The extrema of the y-displacements stay nearly the same with or without structural damping.

With respect to the influence of the SGS model, the following observations can be made:

- The SGS model has no significant influence on the frequency of the FSI phenomenon.

- Variations of the parameter $C_{s}$ of the Smagorinsky model lead to small changes of the maxima of the y-displacements. When $C_{s}$ increases, $\left.U_{y}^{*}\right|_{\max }$ and $\left.U_{y}^{*}\right|_{\min }$ decrease. However, the influence of $C_{s}$ on the FSI results is very limited: Large modifications of $C_{s}$ around the standard value $C_{s}=0.1$ alter the maximal values less than $3 \%$.

- The dynamic Germano model produces extrema of the y-displacements equivalent to those obtained by the Smagorinsky model with $C_{s}=0.065$. The frequency is slightly lower than for the Smagorinsky model with $C_{s}=0.065$ and close to the value reached by the Smagorinsky model with $C_{s}=0.18$.

- The WALE model with $C_{W}=0.33$ generates similar extrema of the y-displacements to those obtained by the Smagorinsky model with $C_{s}=0.1$. The frequency is slightly lower than for the Smagorinsky model with $C_{s}=0.1$. Similar to the dynamic model, the frequency is close to the value achieved by the Smagorinsky model with $C_{s}=0.18$.

Finally, the influence of Young's modulus is the following:

- As expected for a problem involving a plate, when $E_{\text {rubber }}$ increases, $f_{\mathrm{FSI}}$ slowly increases.

- Small changes of $E_{\text {rubber }}$ result in substantial variations of the maxima of the y-displacements.

In summary, the present parameter study shows that Young's modulus $E_{\text {rubber }}$ (or the dimensionless Cauchy number $\mathrm{Cy}_{\text {rubber }}$ ) has the major influence on the FSI phenomenon for the setup used in FSI-PfS-2a. The structural damping has a minor effect and can mainly be neglected. The choice of the SGS model and parameter does not play an important role for FSI-PfS-2a. The classical Smagorinsky model with a standard parameter set to $C_{s}=0.1$ can be used. 


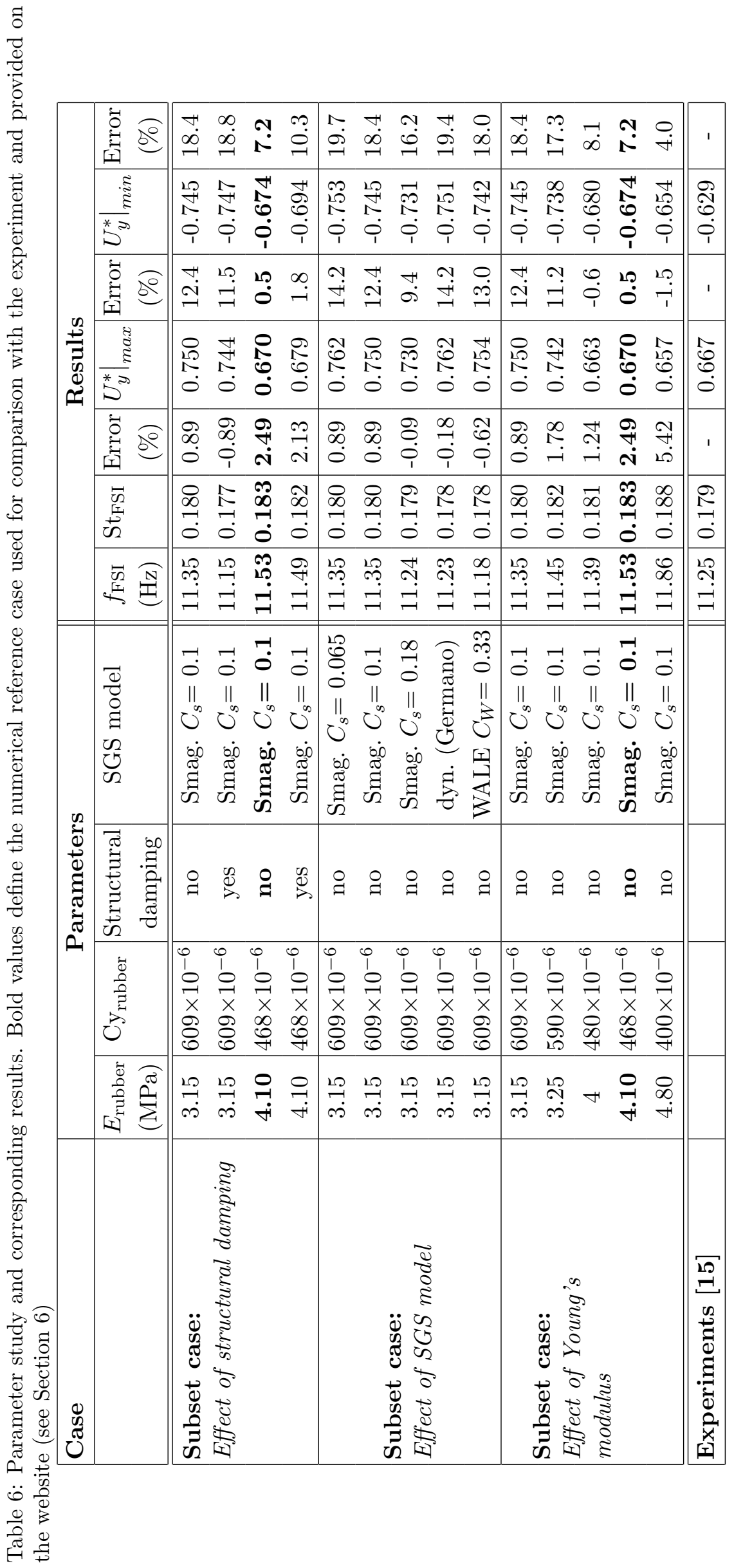




\section{Available Data for Comparison}

The described benchmark FSI-PfS-2a is supposed to test, evaluate and improve numerical FSI codes. Therefore, the authors support all interested groups by the experimental and numerical data presented in this paper. For this purpose the data are made available on the ERCOFTAC Knowledge Base Wiki in the category 'Flow around Bodies' accessible as case 2-14 under

$$
\text { http://qnet-ercoftac.cfms.org.uk/w/index.php/UFR_2-14. }
$$

Available for comparison are:

- The data for the structural test cases described in Kalmbach and Breuer [15].

- The 23 single phase-averaged two-dimensional reference velocity fields of the PIV measurement series used in Section 4 .

- The corresponding numerical data for the same 23 instants in the period.

- The raw and phase-averaged data of the displacement of the flexible structure.

\section{Conclusions}

The current paper presents a numerical investigation of the experimental FSI test case published by Kalmbach and Breuer [15]. It consists of the turbulent flow past a cylinder with a flexible splitter plate and a rear mass, denoted FSI-PfS-2a. The flow is in the so-called subcritical regime $(\mathrm{Re}=30,470)$, which means that due to the transition in the free shear layers the wake is $3 \mathrm{D}$ and chaotic. The flexible structure deforms in the second swiveling mode. However, the large deformations observed are almost 2D due to the steel weight attached behind the flexible rubber plate.

In order to simulate FSI-PfS-2a, a multi-physics code detailed in Breuer et al. [1] is used. Based on the LES method and a modern and accurate shell model, it is especially developed for FSI applications involving turbulent flows around lightweight structures. The computational setup for the CFD and the CSD solver as well as for the FSI coupling is presented in details. Special care is taken of the structure model to approximate the material parameters of the rubber plate. A time interval of two physical seconds of the problem is computed in about 1000 hours wall-clock for a memory requirement of about 22 Gbytes.

The simulation shows a similar unsteady behavior of the flow and the structure as the experiment. However, due to the chaotic nature of turbulence the experimental and numerical results have to be phase-averaged prior to a detailed comparison. The simulated phase-averaged signal fits pretty well to the experimental one concerning form and extrema. Moreover, the frequency of the FSI problem is well predicted with an error of only $2.49 \%$. Six characteristic positions of the structure representing the FSI phenomenon are chosen to compare the phase-averaged numerical flow field with the phase-averaged PIV measurements. For each of these positions the streamwise and transverse velocity components are in very good agreement between the simulation and the experiment. The shedding phenomenon behind the cylinder with the flexible structure and the positions of the vortices convected downstream are correctly predicted.

To better comprehend the current test case a sensitivity study including physical and numerical parameters is carried out leading to the following conclusions: 
- Young's modulus $E_{\text {rubber }}$ has the major influence on the FSI phenomenon for the geometry used in FSI-PfS-2a. Compared to the value provided by Kalmbach and Breuer [15] based on a static structural test, Young's modulus was determined anew based on a more reasonable dynamic structural test. Another parameter study [14] conducted with the geometry of the test case FSI-PfS-1a (similar geometry but other material parameters and no rear mass) has led to the same conclusion.

- Furthermore, in FSI-PfS-2a the structural damping has a minor effect and can also be omitted. This outcome is exactly the contrary to the one found with FSI-PfS-1a. This observation can be explained by the fact that FSI-PfS-1a is an instability-induced excitation and thus more sensitive to structural damping. FSI-PfS-2a, however, is a movement-induced excitation. Here, the damping generated by the viscous fluid exceeds the structural damping by orders of magnitude.

- The sensitivity study also permits to verify that the SGS model and its parameter does not strongly affect the simulation. Indeed, the Smagorinsky model, the dynamic model of Germano and the WALE model deliver similar results for the test case FSI-PfS-2a. This is due to the moderate Reynolds number and the fine grid applied.

The presented test case FSI-PfS-2a is a part of a series of reference test cases designed to validate and improve numerical FSI codes. For each test case all data are available on the ERCOFTAC Knowledge Base Wiki.

\section{Acknowledgments}

The project is financially supported by the Deutsche Forschungsgemeinschaft under the contract number BR 1847/12-1. We gratefully acknowledge the cooperation with the Chair of Structural Analysis of the Technical University Munich providing the codes Carat++ and CoMA, especially Dipl.-Ing. S. Sicklinger, Dr.-Ing. R. Wüchner and Prof. Dr.-Ing. K.U. Bletzinger. The computations were carried out on the German Federal Top-Level Computer SuperMUC at LRZ Munich under the contract number pr47me.

\section{AppendixA. Material parameters for the para-rubber}

In order to experimentally realize the FSI benchmark FSI-PfS-2a presented in Kalmbach and Breuer [15], rubber was the best choice (isotropic mechanical behavior, wide range of usable elastic deformations, etc.). However, rubber is known to have some dynamic characteristics, which are complex to model. Depending on the specific application, a suitable description of the mechanical behavior must be ensured by a more or less complex material model such as Ogden, Neo-Hooke, Mooney-Rivlin or Varga [40]. In FSI-PfS-2a the geometry of the flexible structure consisting of rubber material is chosen to undergo only reversible and moderate elastic deformations. Thus, the simple material law by St. Venant-Kirchhoff can be used in the present study. This decision is also supported by the correct agreement between simulations and measurements obtained on the pure structural test cases presented below. Therefore, just the Poisson's ratio $\nu_{\text {rubber }}$ and Young's modulus $E_{\text {rubber }}$ have to be set. The Poisson's ratio of the para-rubber is $\nu_{\text {rubber }}=0.5$. However, for numerical reasons it has to be set to a slightly different value to avoid dividing by zero (here $\nu_{\text {rubber }}=0.48$ ). Young's modulus $E_{\text {rubber }}$ is hard to determine directly by experiments, but can be approximated based on structural tests and numerical cross-comparison studies as will be shown below. 
As explained in De Nayer et al. [14] for the usual dynamic behavior of rubber, a certain level of damping has to be expected. A simple way to model this damping property is to use the Rayleigh damping: All damping effects are summarized in a velocity proportional damping matrix $\mathbf{D}$ which is composed of a linear combination of the mass matrix $\mathbf{M}$ and the (initial) stiffness matrix $\mathbf{K}_{\mathbf{0}}$ :

$$
\mathbf{D}=\alpha \mathbf{M}+\beta \mathbf{K}_{0} .
$$

The Rayleigh damping factors $\alpha$ and $\beta$ can be evaluated by solving a pair of equations, if two damping ratios and the respective frequencies are known [41]. Since the present FSI phenomenon shows only oscillations in the second swiveling mode, only one damping factor is presently taken into account: The damping factor $\beta$ proportional to the stiffness is approximated, whereas the quantity $\alpha$ proportional to the mass is assumed to be zero. Indeed, the reason for this decision is that the damping proportional to the stiffness is considered to be more realistic (see, e.g., [42]) in this case, since the damping factor $\alpha$ proportional to the mass results in decaying damping effects on higher modes.

In order to determine the material characteristics of the para-rubber (Young's modulus and damping factor), two purely structural tests presented in Kalmbach and Breuer [15] are taken into account: a static test and a decay test. Two different CSD softwares are used in the present investigation: Carat++ and Abaqus $\mathrm{I}^{1}$. Carat++ is the FEM program used within the FSI code. Therefore, it makes sense to perform the material parameter studies based on the same software. Furthermore, the same tests are carried out using the commercial software Abaqus to serve as a reference.

\section{AppendixA.1. Static test}

In the static test the structure (cylinder, plate and rear mass) described in Section 3.1 is arranged in a horizontal alignment. Due to the gravity force, a static deformation of the flexible structure results measured at the tip of the structure. The objective is to approximate the Young's modulus of the para-rubber which is hard to measure directly. Five different Young's moduli $E_{\text {rubber }}$ are evaluated numerically. The results are summarized in Table A.7. Applying Carat++ with $30 \times 10$ shell elements to model the structure, the most appropriate value for Young's modulus $E_{\text {rubber }}$ is found to be about 3.00 MPa. Based on Abaqus and a structure model with $27 \times 30 \mathrm{~S} 8 \mathrm{R}$ shell elements, the best fit for Young's modulus $E_{\text {rubber }}$ is again about $3.00 \mathrm{MPa}$, similar to the value achieved by Carat++. In [15] Ansys Mechanical2 was used for the same purpose with $70 \times 43 \mathrm{D}$-solids elements: The best agreement between the predictions and the measurements was obtained for a Young's modulus $E_{\text {rubber }} \approx 3.15 \mathrm{MPa}$. The Young's modulus approximated by Carat++ and Abaqus using shell elements is about $5 \%$ smaller than the one computed by Ansys using 3D-solid elements.

\section{AppendixA.2. Decay test}

The static test case gives a first approximation of Young's modulus $E_{\text {rubber. }}$. In order to test the para-rubber dynamically, a decay test was carried out. The structure is the same as for the static test, but put in a vertical alignment.

The Young's modulus is set to $E_{\text {rubber }}=3.15 \mathrm{MPa}$ as approximately determined by the static test case. The simulation is again run using Carat++ and Abaqus. The determination of the missing parameters is done in two steps: First, a parametric study on $\beta$ and cross-comparisons

\footnotetext{
${ }^{1}$ http://www.3ds.com/products/simulia/portfolio/abaqus/overview

${ }^{2}$ http://www.ansys.com
} 
Table A.7: Static test case: Three different CSD softwares results compared with the experiment.

\begin{tabular}{|l|c|c|c|r|}
\hline & $E_{\text {rubber }}[\mathrm{MPa}]$ & $y[\mathrm{~m}]$ & $y / D$ & Error $(\%)$ \\
\hline \hline \multirow{3}{*}{ Carat++ } & 2.50 & $-3.588 \mathrm{e}-02$ & -1.63 & 8.53 \\
& 3.00 & $-3.275 \mathrm{e}-02$ & -1.49 & -0.94 \\
& 3.15 & $-3.188 \mathrm{e}-02$ & -1.45 & -3.57 \\
& 3.50 & $-2.999 \mathrm{e}-02$ & -1.36 & -9.29 \\
& 4.00 & $-2.757 \mathrm{e}-02$ & -1.25 & -16.61 \\
\hline \multirow{3}{*}{ Abaqus } & 2.50 & $-3.596 \mathrm{e}-02$ & -1.63 & 8.77 \\
& 3.00 & $-3.283 \mathrm{e}-02$ & -1.49 & -0.70 \\
& 3.15 & $-3.197 \mathrm{e}-02$ & -1.45 & -3.30 \\
& 3.50 & $-3.077 \mathrm{e}-02$ & -1.40 & -6.93 \\
Ansys Mechanical & 4.00 & $-2.766 \mathrm{e}-02$ & -1.26 & -16.33 \\
\hline Kalmbach and Breuer [15]) & 2.50 & $-3.705 \mathrm{e}-02$ & -1.68 & 12.07 \\
& 3.00 & $-3.373 \mathrm{e}-02$ & -1.53 & 2.03 \\
& 3.15 & $-3.282 \mathrm{e}-02$ & -1.49 & -0.73 \\
& 3.50 & $-3.087 \mathrm{e}-02$ & -1.40 & -6.62 \\
& 4.00 & $-2.833 \mathrm{e}-02$ & -1.29 & -14.31 \\
\hline \hline Experiment & & $-3.306 \mathrm{e}-02$ & -1.50 & Ref. \\
\hline
\end{tabular}

with the experimental data leads to $\beta=0.006$. The results are displayed in Fig. 11(a). It can be seen that with this choice of the damping parameter the experimentally observed decay behavior of the oscillation due to the initial deflection is well captured by both CSD softwares. However, the period predicted by Carat ++ and Abaqus $\left(T_{\text {decay_test }_{\text {num }}} \approx 0.280 \mathrm{~s}\right)$ is not in agreement with the experimental one $\left(T_{\text {decay_test }_{\text {exp }}} \approx 0.265 \mathrm{~s}\right)$. The period does not depend on structural damping, but directly on the Young's modulus. Although not directly comparable but the eigenfrequency of an unilaterally clamped beam depends on the square root of the Young's modulus. Thus, a larger Young's modulus yields a higher oscillation frequency. Therefore, in the second step, $E_{\text {rubber }}$ is adjusted in the predictions in order to match the experimental period. The best fit is obtained for $E_{\text {rubber }} \approx 4.1 \mathrm{MPa}$. The results are displayed in Fig. 11(b), The period and the decay rate of the oscillation are now very well predicted by both softwares.

Rubber is known to have a dynamic behavior. The difference in Young's modulus found between the static test case and the decay test is typical. The rubber material becomes stiffer for dynamic deformations. Since the FSI problem is dynamic, the Young's modulus approximated by the decay test is used for the FSI simulation.

[1] M. Breuer, G. De Nayer, M. Münsch, T. Gallinger, R. Wüchner, Fluid-structure interaction using a partitioned semi-implicit predictor-corrector coupling scheme for the application of large-eddy simulation, Journal of Fluids and Structures 29 (2012) 107-130. doi:10.1016/j.jfluidstructs.2011.09.003.

[2] S. Turek, J. Hron, Proposal for numerical benchmarking of fluid-structure interaction between an elastic object and laminar incompressible flow, in: H.-J. Bungartz, M. Schäfer 


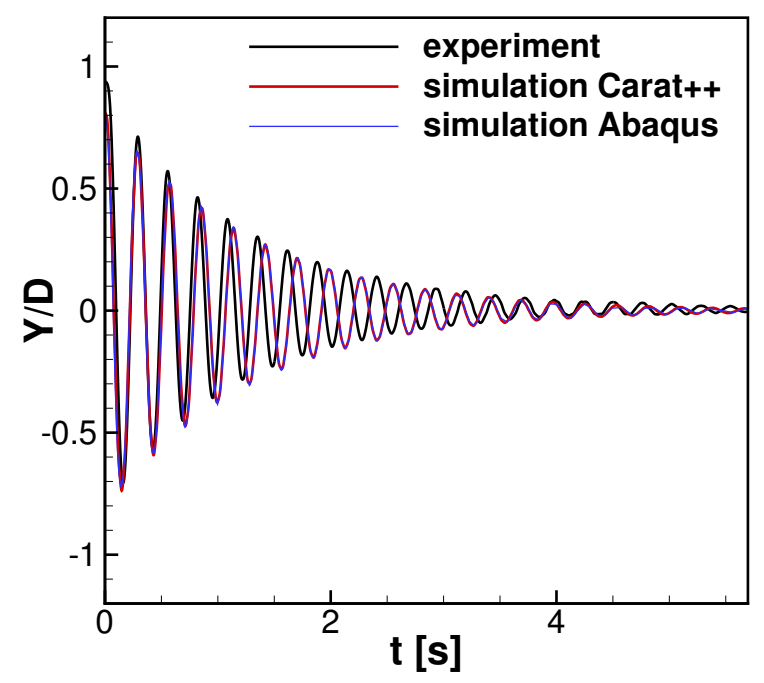

(a) Results with $E_{\text {rubber }}=3.15 \mathrm{MPa}, \alpha=0$ and $\beta=0.006$.

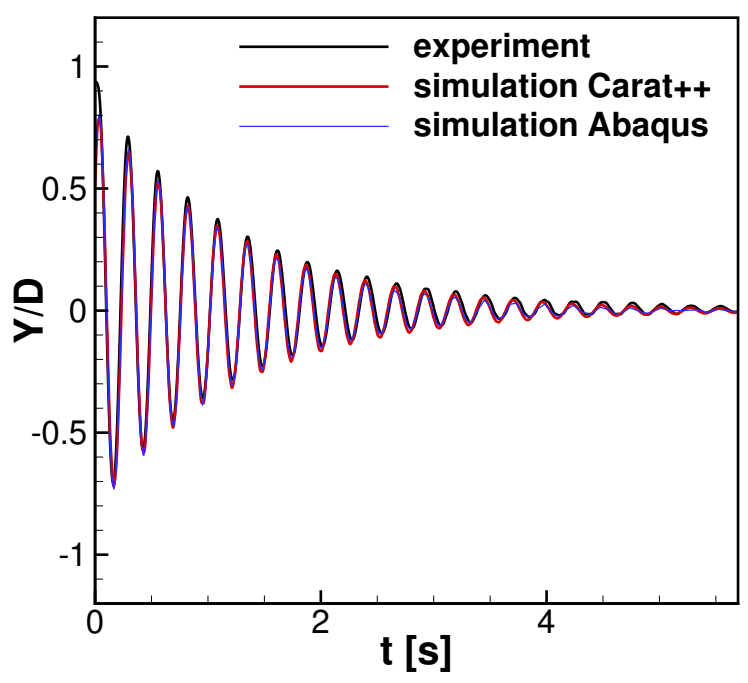

(b) Results with $E_{\text {rubber }}=4.10 \mathrm{MPa}, \alpha=0$ and $\beta=0.006$.

Figure A.11: Decay test: Comparison between structural simulations (Carat++ and Abaqus) and the measured $\mathrm{y}$-deformations of a point in the mid-plane located at a distance of $2 \mathrm{~mm}$ from the shell extremity.

(Eds.), Fluid-Structure Interaction, Vol. 53 of Lecture Notes in Computational Science and Engineering, LNCSE, Springer, Heidelberg, 2006, pp. 371-385.

[3] S. Turek, J. Hron, M. Razzaq, H. Wobker, M. Schäfer, Numerical benchmarking of fluid-structure interaction: A comparison of different discretization and solution approaches, in: H.-J. Bungartz, M. Mehl, M. Schäfer (Eds.), Fluid-Structure Interaction II - Modelling, Simulation, Optimization, Vol. 73 of Lecture Notes in Computational Science and Engineering, LNCSE, Springer, Heidelberg, 2010, pp. 413-424. doi: $10.1007 / 978-3-642-14206-2$.

[4] M. P. Païdoussis, Fluid-Structure Interactions: Slender Structures and Axial Flow, Vol. 2, Academic Press, 2003.

[5] S. Taneda, Waving motions of flags, Journal of the Physical Society of Japan 24 (2) (1968) 392-401. doi:10.1143/JPSJ.24.392.

[6] S. K. Datta, W. G. Gottenberg, Instability of an elastic strip hanging in an airstream, Journal of Applied Mechanics 42 (1975) 195-198.

[7] A. Kornecki, E. H. Dowell, J. O'Brien, On the aeroelastic instability of two-dimensional panels in uniform incompressible flow, Journal of Sound and Vibration 47 (2) (1976) 163-178. doi:http://dx.doi.org/10.1016/0022-460X(76)90715-X.

[8] Y. Watanabe, S. Susuki, M. Sugihara, Y. Sueoka, An experimental study of paper flutter, Journal of Fluids and Structures 16 (4) (2002) 529-542. doi:http://dx.doi.org/10. 1006/jfls.2001.0435.

[9] C. Lemaitre, P. Hémon, E. de Langre, Instability of a long ribbon hanging in axial air flow, Journal of Fluids and Structures 20 (7) (2005) 913-925.

[10] C. Eloy, R. Lagrange, C. Souilliez, L. Schouveiler, Aeroelastic instability of cantilevered flexible plates in uniform flow, Journal of Fluid Mechanics 611 (1) (2008) 97-106. 
[11] J. P. Gomes, H. Lienhart, Experimental benchmark: Self-excited fluid-structure interaction test cases, in: H.-J. Bungartz, M. Mehl, M. Schäfer (Eds.), Fluid-Structure Interaction II - Modelling, Simulation, Optimization, Vol. 73 of Lecture Notes in Computational Science and Engineering, LNCSE, Springer, Heidelberg, 2010, pp. 383-411. doi:10.1007/978-3-642-14206-2.

[12] J. P. Gomes, H. Lienhart, Fluid-structure interaction-induced oscillation of flexible structures in laminar and turbulent flows, Journal of Fluid Mechanics 715 (2013) 537-572.

[13] J. P. Gomes, Fluid-structure interaction-induced oscillation of flexible structures in uniform flows, Ph.D. thesis, Lehrstuhl für Strömungsmechanik, Universität Erlangen-Nürnberg, Germany (2011).

[14] G. De Nayer, A. Kalmbach, M. Breuer, S. Sicklinger, R. Wüchner, Flow past a cylinder with a flexible splitter plate: a complementary experimental-numerical investigation and a new FSI test case (FSI-PfS-1a), Int. Journal of Computers and Fluids 99 (2014) 18-43. doi:10.1016/j.compfluid.2014.04.020.

[15] A. Kalmbach, M. Breuer, Experimental PIV/V3V measurements of vortex-induced fluidstructure interaction in turbulent flow - A new benchmark FSI-PfS-2a, Journal of Fluids and Structures 42 (2013) 369-387. doi:10.1016/j.jfluidstructs.2013.07.004.

[16] E. Naudascher, D. Rockwell, Flow-induced Vibrations: An Engineering Guide, AA Balkema, Rotterdam, Holland, 1994.

[17] F. Durst, M. Schäfer, A parallel block-structured multigrid method for the prediction of incompressible flows, Int. Journal for Numerical Methods in Fluids 22 (6) (1996) 549-565.

[18] F. Durst, M. Schäfer, K. Wechsler, Efficient simulation of incompressible viscous flows on parallel computers, in: E. H. Hirschel (Ed.), Flow Simulation with High-Performance Computers II, Notes on Numerical Fluid Mechanics, Vol. 52(1), Vieweg, 1996, pp. 87-101.

[19] C. M. Rhie, W. L. Chow, Numerical study of the turbulent flow past an airfoil with trailing edge separation, AIAA Journal 21 (11) (1983) 1525-1532.

[20] J. F. Thompson, Z. U. A. Warsi, C. W. Wayne Mastin, Numerical Grid Generation: Foundations and Applications, Vol. 45, North-Holland, 1985.

[21] M. Breuer, Direkte Numerische Simulation und Large-Eddy Simulation turbulenter Strömungen auf Hochleistungsrechnern, Habilitationsschrift, Universität Erlangen-Nürnberg, Berichte aus der Strömungstechnik, Shaker Verlag, Aachen, Germany, 2002.

[22] J. Smagorinsky, General circulation experiments with the primitive equations I: The basic experiment, Monthly Weather Review 91 (3) (1963) 99-165.

[23] M. Germano, U. Piomelli, P. Moin, W. H. Cabot, A dynamic subgrid-scale eddy viscosity model, Physics of Fluids A 3 (1991) 1760-1765.

[24] D. K. Lilly, A proposed modification of the Germano subgrid-scale closure method, Physics of Fluids A 4 (1992) 633-635.

[25] F. Nicoud, F. Ducros, Subgrid-scale stress modelling based on the square of the velocity gradient tensor, Flow, Turbulence and Combustion 62 (3) (1999) 183-200. 
[26] K.-U. Bletzinger, R. Wüchner, A. Kupzok, Algorithmic treatment of shells and free formmembranes in FSI, in: H.-J. Bungartz, M. Schäfer (Eds.), Fluid-Structure Interaction, Vol. 53 of Lecture Notes in Computational Science and Engineering, LNCSE, Springer, Heidelberg, 2006, pp. 336-355.

[27] M. Fischer, M. Firl, H. Masching, K.-U. Bletzinger, Optimization of non-linear structures based on object-oriented parallel programming, in: B. H. V. Topping, J. M. Adam, F. J. Pallarés, R. Bru, M. L. Romero (Eds.), Seventh Int. Conf. Engineering Computational Technology, ECT2010, Civil-Comp Press, Stirlingshire, UK, 2010, p. 67.

[28] R. Wüchner, K.-U. Bletzinger, Stress-adapted numerical form finding of pre-stressed surfaces by the updated reference strategy, Int. Journal for Numerical Methods in Engineering 64 (2) (2005) 143-166.

[29] K.-U. Bletzinger, R. Wüchner, F. Daoud, N. Camprubí, Computational methods for form finding and optimization of shells and membranes, Computer Methods in Applied Mechanics and Engineering 194 (30) (2005) 3438-3452.

[30] F. Dieringer, R. Wüchner, K.-U. Bletzinger, Practical advances in numerical form finding and cutting pattern generation for membrane structures, Journal of the International Association for Shell and Spatial Structures 53 (3) (2012) 147-156.

[31] J. Chung, G. M. Hulbert, A time integration algorithm for structural dynamics with improved numerical dissipation: The generalized- $\alpha$ method, Journal of Applied Mechanics 60 (1993) 371-375.

[32] T. Gallinger, A. Kupzok, U. Israel, K.-U. Bletzinger, R. Wüchner, A computational environment for membrane-wind interaction, in: S. Hartmann, A. Mesiter, M. Schäfer, S. Turek (Eds.), Int. Workshop on Fluid-Structure Interaction: Theory, Numerics and Applications, Kassel University Press GmbH, 2009, pp. 283-294.

[33] C. Farhat, M. Lesoinne, P. LeTallec, Load and motion transfer algorithms for fluidstructure interaction problems with non-matching discrete interfaces: Momentum and energy conservation, optimal discretization and application to aeroelasticity, Computer Methods in Applied Mechanics and Engineering 157 (1998) 95-114.

[34] M. Bischoff, Theorie und Numerik einer dreidimensionalen Schalenformulierung, Ph.D. thesis, Institut für Baustatik, Universität Stuttgart, Germany (1999).

[35] T. Hughes, T. Tezduyar, Finite elements based upon Mindlin plate theory with particular reference to the four-node bilinear isoparametric element, Journal of Applied Mechanics 48 (1981) 587-596.

[36] K. C. Park, G. M. Stanley, A curved $C^{0}$-shell element based on assumed natural-coordinate strains, Journal of Applied Mechanics 53 (1986) 278-290.

[37] M. Bischoff, W. A. Wall, K.-U. Bletzinger, E. Ramm, Models and finite elements for thin-walled structures, in: E. Stein, R. De Borst, T. J. R. Hughes (Eds.), Encyclopedia of Computational Mechanics, Vol. 2, John Wiley \& Sons Ltd, Chichester, 2004, pp. 59-138.

[38] A. Kalmbach, Experimental Investigations of Fluid-Structure Interaction Benchmarks, Ph.D. thesis, Professur für Strömungsmechanik, Helmut-Schmidt Universität Hamburg, Germany (2014). 
[39] E. de Langre, Effects of wind on plants, Annual Review of Fluid Mechanics 40 (1) (2008) 141-168. doi:10.1146/annurev.fluid.40.111406.102135.

[40] G. A. Holzapfel, Nonlinear Solid Mechanics: A Continuum Approach for Engineering, John Wiley \& Sons Ltd., 2000.

[41] R. W. Clough, J. Penzien, Dynamics of Structures, McGraw-Hill, New York, 1993.

[42] W. Krätzig, K. Meskouris, M. Link, Der Ingenieurbau, Baustatik, Baudynamik, Ernst \& Sohn Verlag, Berlin, 1996, Ch. Baudynamik und Systemidentifikation, pp. 365-518. 